\title{
A Mixed Integer Programming Approach to Optimal Feeder Routing for Tree-Based Distribution System: A Case Study
}

\author{
M. Fallah ${ }^{1}$, A. Mohajeri ${ }^{2, *}$, M. Jamshidi ${ }^{3}$
}

\begin{abstract}
A genetic algorithm is proposed to optimize a tree-structured power distribution network considering optimal cable sizing. For minimizing the total cost of the network, a mixed-integer programming model is presented determining the optimal sizes of cables with minimized location-allocation cost. For designing the distribution lines in a power network, the primary factors must be considered as maximum allowable electrical flow in cables, permitted length of cables, maximum permitted voltage drops, and balance of load. The relationship between rates of electric current and cable sizes with consideration of constraints such as voltage drops and length are our essential data. To create a network with a minimum number of arcs and no closed loop such that all the nodes are covered, a minimum spanning tree technique is utilized. Here, we solve the problem using a genetic optimization algorithm and apply the offered approach to a real problem. By comparing the two extracted results from the proposed approach and an exact method, effectiveness of the genetic algorithm for optimization of power distribution network is shown. To demonstrate the validity of the offered model, a case study in Tehran power distribution company in Iran is made.
\end{abstract}

Keywords: Power distribution network, Minimum spanning tree, Location-allocation, distribution transformer, Feeder, Phase

Manuscript was received on 01/06/2017 revised on 05/12/2017 and accepted for publication on 23/12/2017

\section{Introduction}

Electrical energy is a significant source of energy that is so clean. The alternating current (AC) that enters our homes comes from power plants that has huge generators. At a generating plant fuel is burned to boiled water, creating steam that turns turbines and from turning of a generator by turbines, electric energy is produced. The fuel used to heat the water could be fossil fuels like coal, natural gas or oil. In some places wind or water or nuclear power, flowing through hydroelectric dams turns the turbines.

The current produced by a power plant travels to a transformer which increases the voltage. Now it is possible to transmit power over high-voltage transmission lines for long distances and minimum energy loss. Current with high voltage cannot be used by consumers and is too dangerous. That is why it travels on wires often placed high above the ground. To prevent a flow of

\footnotetext{
* Corresponding Author.

${ }^{1}$ Department of Industrial Engineering, Central Tehran Branch, Islamic Azad University, Tehran, Iran, Email: Mohammad.fallah43@yahoo.com

${ }^{2}$ Department of Industrial Engineering, Central Tehran Branch, Islamic Azad University, Tehran, Iran, Email: amir.mohajeri@iauctb.ac.ir

${ }^{3}$ Department of Industrial Engineering, Central Tehran Branch, Islamic Azad University, Tehran, Iran, Email: mahdijamshidi6688@gmail.com
} 
power to the towers or ground, insulators are used. Some factories or businesses that use a significant amount of electricity need a substation specifically for their needs. They require higher voltage and can be fed power directly from the high voltage transmission lines. Most industries need 2400 to 4160 volts to run their massive machinery. Because of their voltage needs, they should have a private substation for their facility. After the power is delivered from the high transmission lines to a specific place, as needed, it reaches another transformer. Here, the voltage is decreased for distribution to customers. Lines with voltages of 33 or 20 kilovolts transfer electricity to distribution transformers which reduce power voltages to 240 volts for general usage in houses, schools and small businesses. When power lines are located overhead, the transformers are usually located on poles near residences or farms. When power lines are situated underground, sizeable green transformer boxes mounted on concrete pads are located throughout neighborhoods. Fig. 1 demonstrates schematically different levels that electric energy overrides from producing phase to consuming phase.

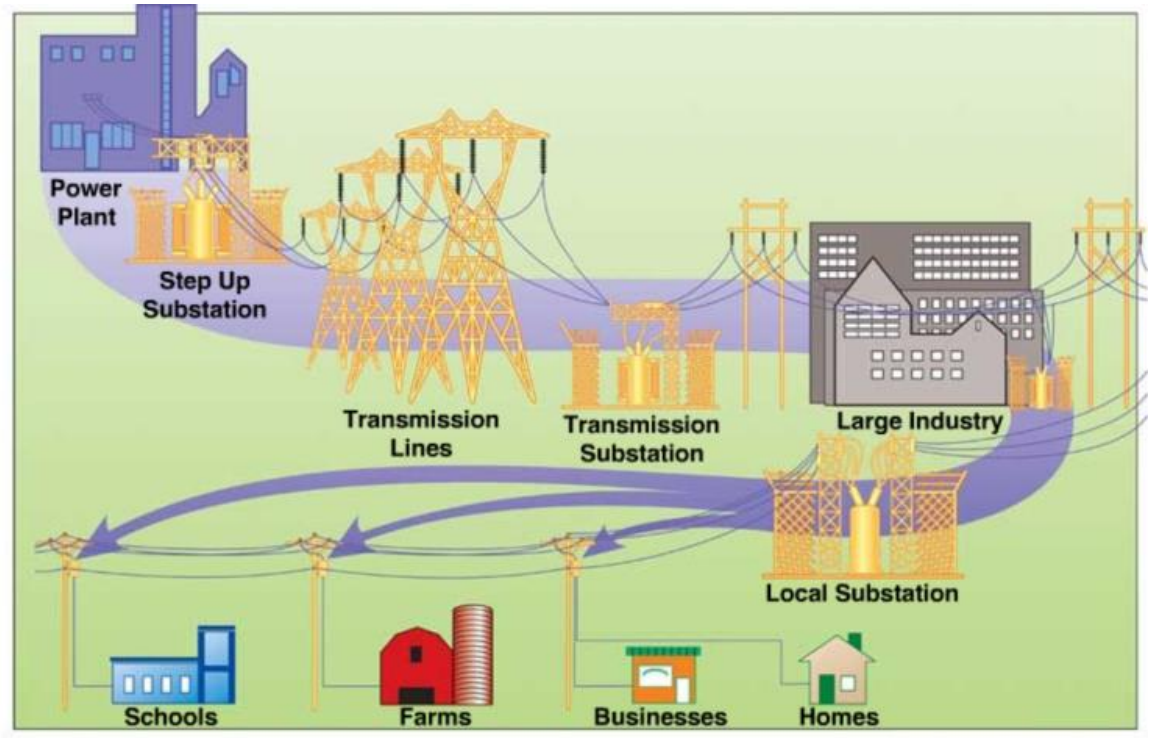

Figure 1. Different phases of production, transmission, and distribution of electric energy

Due to movement of electric energy at different voltage levels over long ways, transmission and distribution planning are very important for the electrical network. To minimize the total cost of an electrical grid, one should have a serious attention to transformer's types and locations and cable sizes. The factors such as cable sizes, voltages, cable lengths have significant effects on the total cost of a power network.

Design of power network distribution lines must be according to the amount of electrical current, length of cables or wires, maximum allowable voltage drops, and power loss. Minimum permissible power voltage in the distribution network is equal to 180 volts for single phase and 380 volts for three stages. In the design of an optimal power distribution network, we should have comprehensive research to consider all significant factors together. Network design in power companies usually is done by heuristic methods which depend on experience and trial and error. Here, we concentrate on power distribution networks and this means that transmitting of electric energy from distribution transformers to consumption zones by cable lines is expected. The maximum allowable voltage drops at load nodes and the maximum load carrying capacity of lines at peak load are two technical restrictions that must be considered for each low voltage feeder. Optimal design of cable sizes has an essential effect on reducing costs in the network. 
We have a set consisting of standard cable sizes made from aluminum and copper with various current transferring capacities that optimal cable will be selected from. In this study, we apply a tree-structured topology for the power distribution problem with the objective function and all the constraints being linear. A tree-structured topology is a graph with a minimum number of arcs, no closed loop and all nodes (consumption zones) being covered in the network. The Minimum Spanning Tree (MST) problem is a combinatorial optimization problem. There is an extensive application of MST in communications, transportation, and power transmission networks. Moreover, indirect applications of MST are network reliability and clustering problems [14 and 15]. On the other hand, MST can be used as a sub-problem in traveling sales man and capacitated MST problems. Pierce [18], Maffoli [13], and Graham and Hell [8] presented comprehensive treatments of the MST problem. Their studies emphasized on traditional algorithms. In the past two decades, much effort conducted for finding faster algorithms using new data structures. For example, we can refer to the undertaken problem by Haymond et al. [10]. Glover et al. [5] and Moret and Shapiro [16] conducted some computational examinations of different kinds of algorithms, and Gavish [4] used these techniques in telecommunication networks. NP-hardness of MST problem was proved by Garey and Johnson [3] and for this reason, heuristic and meta-heuristic algorithms were used in recent years.

Much effort for applying optimization models have been presented recently. Fallah et al. [2] proposed an optimal mathematical model for a green gas network. They designated all active elements on the total cost of a gas network such as pipe diameter, length of pipe, etc. They also identified environmental impact of their proposed gas distribution network with a constraint-based approach to achieve a sustainable development. Nahman and Peric [17] presented a method for optimal planning of radial distribution networks based on a combination of the steepest descent and the simulated annealing approaches. Their goal of the research was the complete network of available routes, and the optimization aim was finding the paths that provided the minimal total cost. They considered factors such as capital recovery, energy loss and undelivered energy costs in their model. Gomez et al. [6] optimized a radial distribution network using ant colony optimization algorithm, and their aim was minimizing the investment and loss costs.

Boulaxis and Papadpoulos [1] applied a dynamic programming technique and geographical information systems. Their network was formed stepwise, so that consumers with fewer distances from the energy source were covered first, and consumers with maximum length were covered next. Goswami [7] utilized a branch exchange technique and determined the initial solution using the minimum spanning tree of the graph of available routes. Then, to satisfy the technical restrictions, the initial solution was reformed considering branch loading and voltage drops with a minimum capital and loss cost. Moreover, he presented a new load flow algorithm that was particularly appropriate for network planning and was not depending on any particular node or branch ordering scheme. To solve the planning problem, some load flows were considered to be equal to the number of successful branch exchanges. Li et al. [12] considered a radial distribution network and reviewed the demand response. Then, they formulated the problem as an optimal power flow problem to maximize the aggregate user utilities and minimizes the supply cost and power line losses, according to the power flow constraints and operating constraints. Zine et al. [21] present a method for decision support enabling the company to make appropriate choices in managing the electricity distribution networks. A multi-criteria formulation was proposed to solve the electricity distribution planning problem, and for multi-criteria problem, they used a transformation method of a multicriteria problem into a problem with a single criterion so that the existence of an optimal solution was guaranteed. 
Here, our objective is to design an optimal power distribution network considering different sizes and types of cables and the locations of the 3-phase transformers. Our original contribution is to present a linear model to design an optimal power distribution network and use a Genetic Algorithm (GA) to solve the proposed power distribution network problem.

Since the 1950s scientists have used artificial intelligence and evolutionary computations and computer programs to simulate natural processes in the real world. Then, in 1953, Nils Barricelli was invited to Princeton to study artificial intelligence. He used the invented digital computer to write software to mimic natural reproduction and mutation. Barricelli's goal was not to solve optimization problems nor to simulate biological evolution, but rather to create artificial life. In 1954, he created the first genetic algorithm work was published in Italian. Barricelli's work was followed in 1957 by Alexander Fraser, a biologist from London. He was the first to use computer programming solely to study evolution [19]. Many biologists followed in his footsteps in the late 1950s and 1960s.

Later, researchers found out that genetic algorithms were useful to find solutions to problems that were computationally hard to solve. Genetic algorithms can simultaneously test many points from all over the solution space, optimize with either discrete or continuous parameters, provide several optimum settings instead of a single solution, and work with many different kinds of data. These advantages allow genetic algorithms to produce stunning results when traditional optimization methods fail miserably [9]. Genetic algorithms do not require extra information (like derivatives) that is unrelated to the values of the possible solutions themselves. The only mechanism that guides the search is the numerical fitness value of the candidate solution, based on the creator's definition of fitness [11]. This approach can be used when the search space is noisy, nonlinear, and derivatives do not even exist. This mechanism also makes the approach applicable in many more situations than the traditional algorithms.

Our results show that our proposed approach has a good accuracy in the obtained solutions and needs less computing time as compared to other methods.

In this study, we conduct a case study of power distribution network in Tehran power distribution company in Iran. Section 2 presents a description of the power distribution network. A mathematical model is given in Section 3. We present our proposed GA algorithm in Section 4. In Section 5, the results of the case study using the real input data are given. Finally, we conclude in Section 6.

\section{Problem Statement}

A schematic configuration of power distribution network is shown in Fig. 2 that consists of the cable lines with different sizes, distribution transformers, and consumers. According to Fig. 2, each transformer is divided into 2 parts of medium voltage and low voltage in which 3 phases with 20000 volts enter the transformer and 3 phases with 400 volts and one null exit from a transformer and enter into a low voltage panel. The voltage between the 2 phases is 380-400 volts, and the voltage between the phase and null is 180-240 volts.

Depending on the transformer capacity, we have some feeders with each feeder containing three different phases and one null. The voltage is a factor that causes the electric current to flow in the cables. The sizes of cables depend on the electrical current of the consumers that flow in the cables. 
In this study, demand of a consumer is assumed to be single phase and in Ampere. Therefore, each consumer receives her needs by a cable consisting of one phase and one null. Here, all the nodes are considered by with a set $\mathrm{V}$ and all arcs are considered to be a set $\mathrm{A}$. In the network, the set of nodes is supposed to designate the consumers and the transformers and the set of arcs is assumed to be the connected cable lines.

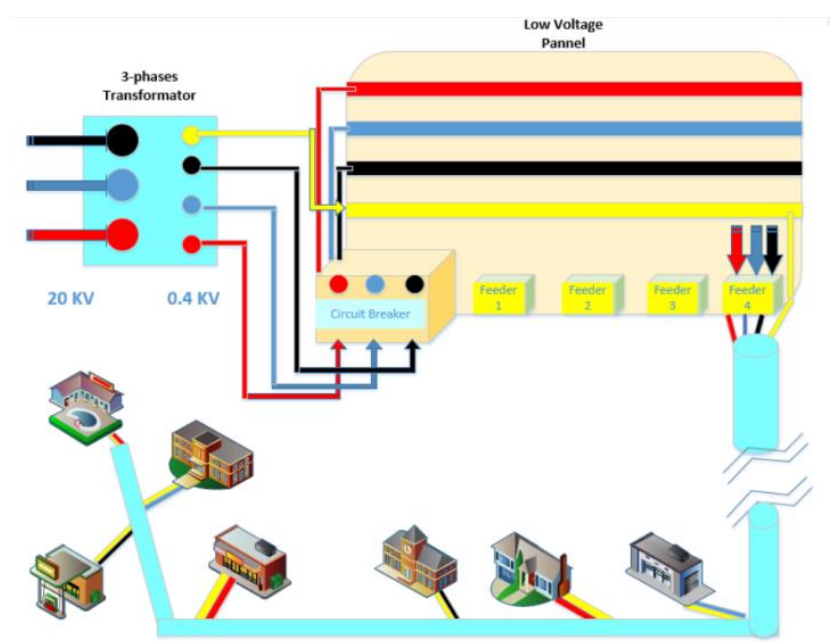

Figure 2. A schematic configuration of a power distribution network

We present a tree-structured power distribution network for our consumption zones and distribution transformers. A connected graph with no closed loop with all nodes covered is called a tree. Determining some appropriate locations of transformers, we utilize expert's opinion. We have three stages in our proposed method:

(a) Designation of types and locations of the distribution transformers.

(b) Determination of each consumer to fed from a feeder and phases of a transformer to keep balance of loads in each phase of the transformer.

(c) Choosing sizes of the cables among the consumers.

In this study, we have two kinds of restrictions, some of which are network infrastructure restrictions, and the others are technical restrictions such as capacity of each phase of the transformer and capacity of each phase of the feeder. Here, we assume that the voltage of power cannot be less than 180 volts in the distribution network. Moreover, considering power loss, length of each feeder cannot be more than 300 meters in the system. In the proposed model, we are to minimize power network's total cost applying the minimum spanning tree (MST) method.

\section{Mathematical Model}

Here, we intend to minimize power network's total cost presenting a mixed-integer linear programming model. We have two kinds of costs in our objective function, constructing cost and cabling cost. Cabling cost is due to three types of cabling: one is medium voltage cabling between two transformers that is constant per unit, the second one is low voltage cabling between the transformer and consumer and the last one is low voltage cabling between two consumers. The low voltage cabling cost depends on the cable size. Here, we use information from the price list of Tehran Power Distribution Company. Table 1 shows the relationship between the amount of electric 
current in Ampere and the cable sizes. In this study, we intend to model a tree-structured power distribution network applying the MST technique and determine the amount of electrical current in network's cable lines. Then, we are to demonstrate how appropriate relationships between the amount of electric current and size of cable can reduce network's cost. Next, we proceed with the elements of the model.

\subsection{Notations}

$I=$ set of candidate sites

$T=$ set of transformers types

$Z=$ set of consumers

$L=$ set of transformers feeders

$K=$ set of transformers phases

$S=$ set of low voltage cables types

\subsection{Parameters}

$c l=$ cost of low voltage cabling per unit distance according to the amount of electric current

$\mathrm{cm}=$ cost of medium voltage cabling per unit distance between the transformers

$c_{t}=$ cost of establishing $t$-type transformer

$g_{z}=$ the single-phase demand of consumer $z$

$n p_{i}=$ maximum number of $t$-type transformer feeders

$Q 1_{t}=$ total capacity of $t$-type transformer in Ampere

$Q 2{ }_{k}=$ each phase of $t$-type transformer capacity in Ampere

$Q 3_{t l k}=$ capacity of phase $k$ of feeder $l$ from $t$-type transformer

$d_{i z}=$ distance between the site of $i$ and consumption zone of $z$

$d^{{ }^{\prime}}{ }_{i i}=$ distance between two transformers

$d_{z z^{\prime}}^{\prime \prime}=$ distance between two consumption zones

$M=$ a big number

\subsection{Decision Variables}

$$
\begin{gathered}
r_{i}=\left\{\begin{array}{l}
1, \text { if } i \text { th site is selected } \\
0, \quad \text { o.w. }
\end{array}\right. \\
h_{i t}= \begin{cases}1, & \text { if } t_{-} \text {type transformer is selected for } i \text { th site } \\
0, & \text { o.w. }\end{cases}
\end{gathered}
$$




$$
\begin{aligned}
& a_{i t l}= \begin{cases}1, & \text { if } l \text { th feeders of } t_{-} \text {type transformer located in } i \text { th site is selected } \\
0, & \text { o. w. }\end{cases} \\
& v_{i t l k}=\left\{\begin{array}{l}
1, \text { if } k \text { th phase of } l \text { th feeder of } t_{-} \text {type transformer located in } i \text { th site is selected } \\
0, \text { o.w. }
\end{array}\right. \\
& y_{i t l k z}=\left\{\begin{array}{l}
1, \text { if } z \text { th consumer is fed from } k \text { th phase of } l \text { th feeder from } t_{-} \text {type } \\
0, \quad \text { o.w. }
\end{array}\right. \\
& w_{z z^{\prime}}= \begin{cases}1, & \text { if there is a direct link between } z \text { th and } z^{\prime} \text { th consumers } \\
0, & \text { o. w. }\end{cases} \\
& u_{i}= \begin{cases}1, & \text { if transformer located in } i \text { th site is a root } \\
0, & \text { o. w. }\end{cases} \\
& N_{z}=\text { number of consumers assigned to consumer } z \\
& f_{i i^{\prime}}=\text { amount of flow between two transformers } i \text { and } i^{\prime} \\
& f_{z z^{\prime}}^{\prime}=\text { amount of flow between two consumers } z \text { and } z^{\prime} \\
& f_{z z^{\prime}}^{\prime \prime}=\text { amount of electric current between two consumers } z \text { and } z^{\prime} \\
& e w_{z}=\text { amount of electric current congested in consumer } z \text { supplied to other consumers } \\
& e w_{i t l k z}^{\prime}=\text { amount of electrical current from } t \text {-type transformer located in site } i \text { to consumer } z \\
& \text { fed from each phase of every feeder } \\
& d w_{z}=\text { cumulative distance of consumer } z \text { from downstream consumers on the same phase } \\
& d w_{i t l k z}^{\prime}=\text { cumulative distance between } t \text {-type transformer located in site } i \text { and the last consumer } \\
& \text { fed from every phase of each feeder } \\
& f d_{z z^{\prime}}=\text { distance between consumer } z \text { and last consumer linked to } z
\end{aligned}
$$

\subsection{Objective Function}

$$
\begin{gathered}
\operatorname{Min}_{0}=f_{1}+f_{2}+f_{3}+f_{4} \\
f_{1}=\sum_{i \in I} \sum_{t \in T} h_{i t} \cdot c_{t}, \\
f_{3}=\sum_{t \in T} \sum_{l \in L} \sum_{k \in K} \sum_{z \in Z} y_{i t l k z} \cdot d_{i z} \cdot c l \\
f_{4}=\sum_{i i^{\prime}} \sum_{i \in Z} \sum_{i i^{\prime}} \cdot c m \\
f_{z^{\prime} \in Z} \\
w_{z z^{\prime}} \cdot d_{z z^{\prime}} \cdot c l .
\end{gathered}
$$

Our objective function consists of four parts minimizing the total cost of the power distribution network. These four components are described next: 
$f_{1}$ : The cost of establishing transformers in sites

$f_{2}$ : The cost of power distribution from transformers to consumers

$f_{3}$ : The cost of power distribution among the transformers

$f_{4}$ : The cost of power distribution among the consumers

According to Table 1, we have 11 kinds of low voltage cables with different capacities and prices. So, instead of second part of the objective function, $\mathrm{f}_{2}$, we can have the following objective function:

$$
\begin{aligned}
& \sum_{i \in I} \sum_{t \in T} \sum_{l \in L} \sum_{k \in K} \sum_{z \in Z} y 1_{i t l k z} \times d_{i z} \times(108000) \\
+ & \sum_{i \in I} \sum_{t \in T} \sum_{l \in L} \sum_{k \in K} \sum_{z \in Z} y 2_{i t l k z} \times d_{i z} \times(163000) \\
+ & \sum_{i \in I} \sum_{t \in T} \sum_{l \in L} \sum_{k \in K} \sum_{z \in Z} y 3_{i t l k z} \times d_{i z} \times(195000) \\
+ & \sum_{i \in I} \sum_{t \in T} \sum_{l \in L} \sum_{k \in K} \sum_{z \in Z} y 4_{i t l k z} \times d_{i z} \times(276500) \\
+ & \sum_{i \in I} \sum_{t \in T} \sum_{l \in L} \sum_{k \in K} \sum_{z \in Z} y 5_{i t l k z} \times d_{i z} \times(354800) \\
+ & \sum_{i \in I} \sum_{t \in T} \sum_{l \in L} \sum_{k \in K} \sum_{z \in Z} y 6_{i t l k z} \times d_{i z} \times(464000) \\
+ & \sum_{i \in I} \sum_{t \in T} \sum_{l \in L} \sum_{k \in K} \sum_{z \in Z} y 7_{i t l k z} \times d_{i z} \times(627900) \\
+ & \sum_{i \in I} \sum_{t \in T} \sum_{l \in L} \sum_{k \in K} \sum_{z \in Z} y 8_{i t l k z} \times d_{i z} \times(839900) \\
+ & +\sum_{i \in I} \sum_{t \in T} \sum_{i \in I} \sum_{k \in K} \sum_{t \in T} y 9_{i t l k z} \times d_{i z} \times(1120600) \\
+ & \sum_{i \in I} \sum_{t \in T} \sum_{l \in L} \sum_{k \in K} \sum_{z \in Z} y 10_{i t l k z} \times d_{i z} \times(1287100) \\
& y 11_{i t l k z} \times d_{i z} \times(1647100)
\end{aligned}
$$

where the binary variables of $y 1_{i t l k z}, \ldots, y 11_{\text {itlkz }}$ are used to create a link between the amount of electric current with the corresponding cost of the cables being used among the transformers and consumers. Likewise, the forth part of the objective function, $\mathrm{f}_{4}$, should be altered as follows: 


$$
\begin{aligned}
& \sum_{z \in Z} \sum_{z^{\prime} \in Z} w 1_{z z^{\prime}} \times d_{z z^{\prime}}^{\prime \prime} \times(108000) \\
& +\sum_{z \in Z} \sum_{z^{\prime} \in Z} w 2_{z z^{\prime}} \times d_{z z^{\prime}}^{\prime \prime} \times(163000) \\
& +\sum_{z \in Z} \sum_{z^{\prime} \in Z} w 3_{z z^{\prime}} \times d_{z z^{\prime}}^{\prime \prime} \times(195000) \\
& +\sum_{z \in Z} \sum_{z^{\prime} \in Z} w 4_{z z^{\prime}} \times d_{z z^{\prime}}^{\prime \prime} \times(276500) \\
& +\sum_{z \in Z} \sum_{z^{\prime} \in Z} w 5_{z z^{\prime}} \times d_{z z^{\prime}}^{\prime \prime} \times(354800) \\
& +\sum_{z \in Z} \sum_{z^{\prime} \in Z} w 6_{z z^{\prime}} \times d_{z z^{\prime}}^{\prime \prime} \times(464000) \\
& +\sum_{z \in Z} \sum_{z^{\prime} \in Z} w 7_{z z^{\prime}} \times d_{z z^{\prime}}^{\prime \prime} \times(627900) \\
& +\sum_{z \in Z} \sum_{z^{\prime} \in Z} w 8_{z z^{\prime}} \times d_{z z^{\prime}}^{\prime \prime} \times(839900) \\
& +\sum_{z \in Z} \sum_{z^{\prime} \in Z} w 9_{z z^{\prime}} \times d_{z z^{\prime}}^{\prime \prime} \times(1120600) \\
& +\sum_{z \in Z} \sum_{z_{z^{\prime}} \in Z} w 10_{z z^{\prime}} \times d_{z z^{\prime}} \times(1287100) \\
& +\sum_{z z^{\prime}} \times d_{z z^{\prime}}^{\prime \prime} \times(1647100)
\end{aligned}
$$

\subsection{Constraints}

\subsubsection{Structural Constraints (Tree-Structured Network)}

$$
\begin{gathered}
u_{i} \leq r_{i}, \quad \forall i \in I, \\
\sum_{i \in I} u_{i}=1, \\
r_{i}-M\left(r_{i^{\prime}}-1\right) \geq x_{i i^{\prime}} \quad \forall i, i^{\prime} \in I, \\
r_{i^{\prime}} \geq x_{i i^{\prime}}, \quad \forall i, i^{\prime} \in I
\end{gathered}
$$




$$
\begin{aligned}
& \sum_{i \in I} x_{i i^{\prime}} \geq\left(1-u_{i^{\prime}}\right)+M\left(r_{i^{\prime}}-1\right), \quad \forall i^{\prime} \in I, \\
& \sum_{i \in I} x_{i i^{\prime}} \leq\left(1-u_{i^{\prime}}\right)-M\left(r_{i^{\prime}}-1\right), \quad \forall i^{\prime} \in I, \\
& \sum_{i \in I} x_{i i^{\prime}} \leq r_{i^{\prime}}, \quad \forall i^{\prime} \in I \\
& x_{i i^{\prime}} \leq f_{i i^{\prime}}, \quad \forall i, i^{\prime} \in I \text {, } \\
& f_{i i^{\prime}} \leq x_{i i^{\prime}} M, \quad \forall i, i^{\prime} \in I \\
& \sum_{i \in I} f_{i i^{\prime}}-\sum_{i^{\prime} \in I} f_{i^{\prime} i^{\prime \prime}} \geq\left(\left(-u_{i^{\prime}} M\right)+1\right)+\left(r_{i^{\prime}}-1\right), \forall i^{\prime} \in I \text {, } \\
& \sum_{i \in I} f_{i i^{\prime}}-\sum_{i^{\prime} \in I} f_{i^{\prime} i^{\prime \prime}} \leq\left(\left(u_{i^{\prime}} M\right)+1\right)+\left(r_{i^{\prime}}-1\right), \forall i^{\prime} \in I, \\
& \sum_{l \in L} a_{i t l} \geq h_{i t}, \quad \forall i \in I, \forall t \in T, \\
& \sum_{l \in L} a_{i t l} \leq h_{i t} \cdot n p_{t}, \quad \forall i \in I, \forall t \in T, \\
& \sum_{k \in K} v_{i t l k}=3 a_{i t l}, \quad \forall i \in I, \forall t \in T, \forall l \in L, \\
& \sum_{z \in Z} y_{i t l k z}=v_{i t l k}, \quad \forall i \in I, \forall t \in T, \forall l \in L, k \in K, \\
& \sum_{z \in Z} w_{z z^{\prime}}+\sum_{i \in I} \sum_{t \in T} \sum_{l \in L} \sum_{k \in K} y_{i t l k z^{\prime}}=1, \forall z^{\prime} \in Z, \\
& N_{z}-\sum_{z^{\prime} \in Z} f_{z z^{\prime}}^{\prime}=1, \quad \forall z \in Z, \\
& N_{z^{\prime}}+M\left(w_{z z^{\prime}}-1\right) \leq f_{z z^{\prime}}^{\prime}, \quad \forall z, z^{\prime} \in Z \text {, } \\
& N_{z^{\prime}}-M\left(w_{z z^{\prime}}-1\right) \geq f_{z z^{\prime}}^{\prime}, \quad \forall z, z^{\prime} \in Z \text {, } \\
& f_{z z^{\prime}}^{\prime} \leq w_{z z^{\prime}} M, \quad \forall z, z^{\prime} \in Z,
\end{aligned}
$$




$$
f_{z z^{\prime}}^{\prime} \geq w_{z z^{\prime}}, \quad \forall z, z^{\prime} \in Z
$$

Constraint (4) expresses that an active site can only be a root. Constraint (5) certifies that the number of network's root is equal to one. The link between two transformers is presented in constraints (6) and (7). Constraints (8)-(10) indicate that if a transformer is not the root, then it can have just one link with other transformers. Constraints (11) and (12) show the amount of flow between $i$ and $i^{\prime}$ transformers. Constraints (13) and (14) ensure that the network is free of a closed loop. The number of feeders of each transformer is imposed by constraints (15) and (16). Constraint (17) imposes that each feeder has exactly 3 phases. Constraint (18) imposes that precisely one consumer can directly connect to a phase of each feeder of the transformer. Constraint (19) imposes that each consumer for receiving service has two ways, either from another consumer or from a transformer. The number of consumers assigned to consumer $z$ is shown by constraint (20). The flow between two consumers is presented by constraints (21)-(24).

\subsubsection{Site Constraint}

$$
\sum_{t \in T} h_{i t}=r_{i}, \quad \forall i \in I
$$

which expresses that in each site we can have just one type of transformer for providing service to consumers.

\subsubsection{Electric Current and Cable Size Constraints}

$$
\begin{gathered}
\left(w_{z z^{\prime}}-1\right) M+\left(g_{z^{\prime}}+e w_{z^{\prime}}\right) \leq f_{z z^{\prime}}^{\prime \prime}, \quad \forall z, z^{\prime} \in Z, \\
\left(w_{z z^{\prime}}-1\right)(-M)+\left(g_{z^{\prime}}+e w_{z^{\prime}}\right) \geq f_{z z^{\prime}}^{\prime \prime}, \forall z, z^{\prime} \in Z, \\
f_{z z^{\prime}}^{\prime \prime} \leq w_{z z^{\prime}} M, \quad \forall z, z^{\prime} \in Z, \\
f_{z z^{\prime}}^{\prime \prime} \geq w_{z z^{\prime}}, \quad \forall z, z^{\prime} \in Z \\
\sum_{z z^{\prime} \in Z} f_{z z^{\prime}}^{\prime \prime}=e w_{z}, \quad \forall z \in Z, \\
\begin{array}{c}
\left.w 1_{z z^{\prime}}\right)+\left(1-m 1_{z z^{\prime}}\right) M \\
+\left(m 2_{z z^{\prime}}+m 3_{z z^{\prime}}+m 4_{z z^{\prime}}+m 5_{z z^{\prime}}+m 6_{z z^{\prime}}+m 7_{z z^{\prime}}+m 8_{z z^{\prime}}+m 9_{z z^{\prime}}\right. \\
\left.+m 10_{z z^{\prime}}+m 1_{z z^{\prime}}\right),
\end{array}
\end{gathered}
$$




$$
\begin{aligned}
& f_{z z^{\prime}}^{\prime \prime} \geq\left(w 1_{z z^{\prime}}\right)-\left(1-m 1_{z z^{\prime}}\right) M \\
& -\left(m 2_{z z^{\prime}}+m 3_{z z^{\prime}}+m 4_{z z^{\prime}}+m 5_{z z^{\prime}}+m 6_{z z^{\prime}}+m 7_{z z^{\prime}}+m 8_{z z^{\prime}}+m 9_{z z^{\prime}}\right. \\
& \left.+m 10_{z z^{\prime}}+m 11_{z z^{\prime}}\right) \\
& f_{z z^{\prime}}^{\prime \prime} \leq\left(45 \times w 2_{z z^{\prime}}\right)+\left(1-m 2_{z z^{\prime}}\right) M \\
& +\left(m 1_{z z^{\prime}}+m 3_{z z^{\prime}}+m 4_{z z^{\prime}}+m 5_{z z^{\prime}}+m 6_{z z^{\prime}}+m 7_{z z^{\prime}}+m 8_{z z^{\prime}}+m 9_{z z^{\prime}}\right. \\
& \left.+m 10_{z z^{\prime}}+m 11_{z z^{\prime}}\right) \text {, } \\
& f_{z z^{\prime}}^{\prime \prime} \geq\left(31 \times w 2_{z z^{\prime}}\right)-\left(1-m 2_{z z^{\prime}}\right) M \\
& -\left(m 1_{z z^{\prime}}+m 3_{z z^{\prime}}+m 4_{z z^{\prime}}+m 5_{z z^{\prime}}+m 6_{z z^{\prime}}+m 7_{z z^{\prime}}+m 8_{z z^{\prime}}+m 9_{z z^{\prime}}\right. \\
& \left.+m 10_{z z^{\prime}}+m 11_{z z^{\prime}}\right) \text {, } \\
& f_{z z^{\prime}}^{\prime \prime} \leq\left(70 \times w 3_{z z^{\prime}}\right)+\left(1-m 3_{z z^{\prime}}\right) M \\
& +\left(m 1_{z z^{\prime}}+m 2_{z z^{\prime}}+m 4_{z z^{\prime}}+m 5_{z z^{\prime}}+m 6_{z z^{\prime}}+m 7_{z z^{\prime}}+m 8_{z z^{\prime}}+m 9_{z z^{\prime}}\right. \\
& \left.+m 10_{z z^{\prime}}+m 11_{z z^{\prime}}\right) \text {, } \\
& f_{z z^{\prime}}^{\prime \prime} \geq\left(46 \times w 3_{z z^{\prime}}\right)-\left(1-m 3_{z z^{\prime}}\right) M \\
& -\left(m 1_{z z^{\prime}}+m 2_{z z^{\prime}}+m 4_{z z^{\prime}}+m 5_{z z^{\prime}}+m 6_{z z^{\prime}}+m 7_{z z^{\prime}}+m 8_{z z^{\prime}}+m 9_{z z^{\prime}}\right. \\
& \left.+m 10_{z z^{\prime}}+m 11_{z z^{\prime}}\right) \text {, } \\
& f_{z z^{\prime}}^{\prime \prime} \leq\left(110 \times w 4_{z z^{\prime}}\right)+\left(1-m 4_{z z^{\prime}}\right) M \\
& +\left(m 1_{z z^{\prime}}+m 2_{z z^{\prime}}+m 3_{z z^{\prime}}+m 5_{z z^{\prime}}+m 6_{z z^{\prime}}+m 7_{z z^{\prime}}+m 8_{z z^{\prime}}+m 9_{z z^{\prime}}\right. \\
& \left.+m 10_{z z^{\prime}}+m 11_{z z^{\prime}}\right) \text {, } \\
& f_{z z^{\prime}}^{\prime \prime} \geq\left(71 \times w 4_{z z^{\prime}}\right)-\left(1-m 4_{z z^{\prime}}\right) M \\
& -\left(m 1_{z z^{\prime}}+m 2_{z z^{\prime}}+m 3_{z z^{\prime}}+m 5_{z z^{\prime}}+m 6_{z z^{\prime}}+m 7_{z z^{\prime}}+m 8_{z z^{\prime}}+m 9_{z z^{\prime}}\right. \\
& \left.+m 10_{z z^{\prime}}+m 11_{z z^{\prime}}\right) \text {, } \\
& f_{z z^{\prime}}^{\prime \prime} \leq\left(133 \times w 5_{z z^{\prime}}\right)+\left(1-m 5_{z z^{\prime}}\right) M \\
& +\left(m 1_{z z^{\prime}}+m 2_{z z^{\prime}}+m 3_{z z^{\prime}}+m 4_{z z^{\prime}}+m 6_{z z^{\prime}}+m 7_{z z^{\prime}}+m 8_{z z^{\prime}}+m 9_{z z^{\prime}}\right. \\
& \left.+m 10_{z z^{\prime}}+m 11_{z z^{\prime}}\right) \\
& f_{z z^{\prime}}^{\prime \prime} \geq\left(111 \times w 5_{z z^{\prime}}\right)-\left(1-m 5_{z z^{\prime}}\right) M \\
& -\left(m 1_{z z^{\prime}}+m 2_{z z^{\prime}}+m 3_{z z^{\prime}}+m 4_{z z^{\prime}}+m 6_{z z^{\prime}}+m 7_{z z^{\prime}}+m 8_{z z^{\prime}}+m 9_{z z^{\prime}}\right. \\
& \left.+m 10_{z z^{\prime}}+m 11_{z z^{\prime}}\right) \\
& f_{z z^{\prime}}^{\prime \prime} \leq\left(157 \times w 6_{z z^{\prime}}\right)+\left(1-m 6_{z z^{\prime}}\right) M \\
& +\left(m 1_{z z^{\prime}}+m 2_{z z^{\prime}}+m 3_{z z^{\prime}}+m 4_{z z^{\prime}}+m 5_{z z^{\prime}}+m 7_{z z^{\prime}}+m 8_{z z^{\prime}}+m 9_{z z^{\prime}}\right. \\
& \left.+m 10_{z z^{\prime}}+m 11_{z z^{\prime}}\right) \text {, } \\
& f_{z z^{\prime}}^{\prime \prime} \geq\left(134 \times w 6_{z z^{\prime}}\right)-\left(1-m 6_{z z^{\prime}}\right) M \\
& -\left(m 1_{z z^{\prime}}+m 2_{z z^{\prime}}+m 3_{z z^{\prime}}+m 4_{z z^{\prime}}+m 5_{z z^{\prime}}+m 7_{z z^{\prime}}+m 8_{z z^{\prime}}+m 9_{z z^{\prime}}\right. \\
& \left.+m 10_{z z^{\prime}}+m 11_{z z^{\prime}}\right) \text {, } \\
& f_{z z^{\prime}}^{\prime \prime} \leq\left(195 \times w 7_{z z^{\prime}}\right)+\left(1-m 7_{z z^{\prime}}\right) M \\
& +\left(m 1_{z z^{\prime}}+m 2_{z z^{\prime}}+m 3_{z z^{\prime}}+m 4_{z z^{\prime}}+m 5_{z z^{\prime}}+m 6_{z z^{\prime}}+m 8_{z z^{\prime}}+m 9_{z z^{\prime}}\right. \\
& \left.+m 10_{z z^{\prime}}+m 11_{z z^{\prime}}\right) \\
& f_{z z^{\prime}}^{\prime \prime} \geq\left(158 \times w 7_{z z^{\prime}}\right)-\left(1-m 7_{z z^{\prime}}\right) M \\
& -\left(m 1_{z z^{\prime}}+m 2_{z z^{\prime}}+m 3_{z z^{\prime}}+m 4_{z z^{\prime}}+m 5_{z z^{\prime}}+m 6_{z z^{\prime}}+m 8_{z z^{\prime}}+m 9_{z z^{\prime}}\right. \\
& \left.+m 10_{z z^{\prime}}+m 11_{z z^{\prime}}\right) \text {, } \\
& f_{z z^{\prime}}^{\prime \prime} \leq\left(233 \times w 8_{z z^{\prime}}\right)+\left(1-m 8_{z z^{\prime}}\right) M \\
& +\left(m 1_{z z^{\prime}}+m 2_{z z^{\prime}}+m 3_{z z^{\prime}}+m 4_{z z^{\prime}}+m 5_{z z^{\prime}}+m 6_{z z^{\prime}}+m 7_{z z^{\prime}}+m 9_{z z^{\prime}}\right. \\
& \left.+m 10_{z z^{\prime}}+m 11_{z z^{\prime}}\right) \\
& f_{z z^{\prime}}^{\prime \prime} \geq\left(196 \times w 8_{z z^{\prime}}\right)-\left(1-m 8_{z z^{\prime}}\right) M \\
& -\left(m 1_{z z^{\prime}}+m 2_{z z^{\prime}}+m 3_{z z^{\prime}}+m 4_{z z^{\prime}}+m 5_{z z^{\prime}}+m 6_{z z^{\prime}}+m 7_{z z^{\prime}}+m 9_{z z^{\prime}}\right. \\
& \left.+m 10_{z z^{\prime}}+m 11_{z z^{\prime}}\right) \text {, }
\end{aligned}
$$




$$
\begin{aligned}
f_{z z^{\prime}}^{\prime \prime} \leq(265 \times & \left.w 9_{z z^{\prime}}\right)+\left(1-m 9_{z z^{\prime}}\right) M \\
& +\left(m 1_{z z^{\prime}}+m 2_{z z^{\prime}}+m 3_{z z^{\prime}}+m 4_{z z^{\prime}}+m 5_{z z^{\prime}}+m 6_{z z^{\prime}}+m 7_{z z^{\prime}}+m 8_{z z^{\prime}}\right. \\
& \left.+m 1_{z z^{\prime}}+m 1_{z z^{\prime}}\right), \\
f_{z z^{\prime}}^{\prime \prime} \geq(234 \times & \left.w 9_{z z^{\prime}}\right)-\left(1-m 9_{z z^{\prime}}\right) M \\
& -\left(m 1_{z z^{\prime}}+m 2_{z z^{\prime}}+m 3_{z z^{\prime}}+m 4_{z z^{\prime}}+m 5_{z z^{\prime}}+m 6_{z z^{\prime}}+m 7_{z z^{\prime}}+m 8_{z z^{\prime}}\right. \\
& \left.+m 10_{z z^{\prime}}+m 1_{z z^{\prime}}\right), \\
f_{z z^{\prime}}^{\prime \prime} \leq(297 \times & \left.w 10_{z z^{\prime}}\right)+\left(1-m 10_{z z^{\prime}}\right) M \\
& +\left(m 1_{z z^{\prime}}+m 2_{z z^{\prime}}+m 3_{z z^{\prime}}+m 4_{z z^{\prime}}+m 5_{z z^{\prime}}+m 6_{z z^{\prime}}+m 7_{z z^{\prime}}+m 8_{z z^{\prime}}\right. \\
& \left.+m 9_{z z^{\prime}}+m 11_{z z^{\prime}}\right), \\
f_{z z^{\prime}}^{\prime \prime} \geq(266 \times & \left.w 10_{z z^{\prime}}\right)-\left(1-m 10_{z z^{\prime}}\right) M \\
& -\left(m 1_{z z^{\prime}}+m 2_{z z^{\prime}}+m 3_{z z^{\prime}}+m 4_{z z^{\prime}}+m 5_{z z^{\prime}}+m 6_{z z^{\prime}}+m 7_{z z^{\prime}}+m 8_{z z^{\prime}}\right. \\
& \left.+m 9_{z z^{\prime}}+m 11_{z z^{\prime}}\right), \\
f_{z z^{\prime}}^{\prime \prime} \leq(334 \times & \left.w 11_{z z^{\prime}}\right)+\left(1-m 11_{z z^{\prime}}\right) M \\
& +\left(m 1_{z z^{\prime}}+m 2_{z z^{\prime}}+m 3_{z z^{\prime}}+m 4_{z z^{\prime}}+m 5_{z z^{\prime}}+m 6_{z z^{\prime}}+m 7_{z z^{\prime}}+m 8_{z z^{\prime}}\right. \\
& \left.+m 9_{z z^{\prime}}+m 10_{z z^{\prime}}\right), \\
f_{z z^{\prime}}^{\prime \prime} \geq(298 \times & \left.w 11_{z z^{\prime}}\right)-\left(1-m 11_{z z^{\prime}}\right) M \\
& -\left(m 1_{z z^{\prime}}+m 2_{z z^{\prime}}+m 3_{z z^{\prime}}+m 4_{z z^{\prime}}+m 5_{z z^{\prime}}+m 6_{z z^{\prime}}+m 7_{z z^{\prime}}+m 8_{z z^{\prime}}\right. \\
& \left.+m 9_{z z^{\prime}}+m 10_{z z^{\prime}}\right), \\
m 1_{z z^{\prime}}+m 2_{z z^{\prime}} & +m 3_{z z^{\prime}}+m 4_{z z^{\prime}}+m 5_{z z^{\prime}}+m 6_{z z^{\prime}}+m 7_{z z^{\prime}}+m 8_{z z^{\prime}}+m 9_{z z^{\prime}}+m 10_{z z^{\prime}} \\
& +m 11_{z z^{\prime}}=w w_{z z^{\prime}} \forall z, z^{\prime} \in Z
\end{aligned}
$$

$\left(y_{i t l k z}-1\right)(-M)+\left(g_{z}+e w_{z}\right) \geq e w_{i t l k z}^{\prime}, \quad \forall i \in I, \forall t \in T, \forall l \in L, \forall k \in K, \forall z \in Z$,

$e w_{i t l k z}^{\prime} \leq M y_{i t l k z}, \forall i \in I, \forall t \in T, \forall l \in L, k \in K, z \in Z$

$e w_{i t l k z}^{\prime} \geq y_{i t l k z}, \forall i \in I, \forall t \in T, \forall l \in L, k \in K, z \in Z$,

$$
\begin{aligned}
& e w_{i t l k z}^{\prime} \leq(30 \times\left.y 1_{i t l k z}\right)+\left(1-n 1_{i t l k z}\right) M \\
&+\left(n 2_{i t l k z}+n 3_{i t l k z}+n 4_{i t l k z}+n 5_{i t l k z}+n 6_{i t l k z}+n 7_{i t l k z}+n 8_{i t l k z}\right. \\
&\left.+n 9_{i t l k z}+n 10_{i t l k z}+n 11_{i t l k z}\right) M \\
& e w_{i t l k z}^{\prime} \geq\left(y 1_{i t l k z}\right)-\left(1-n 1_{i t l k z}\right) M \\
&-\left(n 2_{i t l k z}+n 3_{i t l k z}+n 4_{i t l k z}+n 5_{i t l k z}+n 6_{i t l k z}+n 7_{i t l k z}+n 8_{i t l k z}\right. \\
&\left.+n 9_{i t l k z}+n 10_{i t l k z}+n 11_{i t l k z}\right) M,
\end{aligned}
$$

$e w_{i t l k z}^{\prime} \leq\left(45 \times y 2_{i t l k z}\right)+\left(1-n 2_{i t l k z}\right) M$

$+\left(n 1_{i t l k z}+n 3_{i t l k z}+n 4_{i t l k z}+n 5_{i t l k z}+n 6_{i t l k z}+n 7_{i t l k z}+n 8_{i t l k z}\right.$ $\left.+n 9_{i t l k z}+n 10_{i t l k z}+n 11_{i t l k z}\right) M$,

$e w_{i t l k z}^{\prime} \geq\left(31 \times y 2_{i t l k z}\right)-\left(1-n 2_{i t l k z}\right) M$

$$
\begin{aligned}
& -\left(n 1_{i t l k z}+n 3_{i t l k z}+n 4_{i t l k z}+n 5_{i t l k z}+n 6_{i t l k z}+n 7_{i t l k z}+n 8_{i t l k z}\right. \\
& \left.+n 9_{i t l k z}+n 10_{i t l k z}+n 11_{i t l k z}\right) M
\end{aligned}
$$




$$
\begin{aligned}
e w_{i t l k z}^{\prime} \leq(70 \times & \left.y 3_{i t l k z}\right)+\left(1-n 3_{i t l k z}\right) M \\
& +\left(n 1_{i t l k z}+n 2_{i t l k z}+n 4_{i t l k z}+n 5_{i t l k z}+n 6_{i t l k z}+n 7_{i t l k z}+n 8_{i t l k z}\right. \\
& \left.+n 9_{i t l k z}+n 10_{i t l k z}+n 11_{i t l k z}\right) M,
\end{aligned}
$$

$e w_{i t l k z}^{\prime} \geq\left(46 \times y 3_{i t l k z}\right)-\left(1-n 3_{i t l k z}\right) M$

$-\left(n 1_{i t l k z}+n 2_{i t l k z}+n 4_{i t l k z}+n 5_{i t l k z}+n 6_{i t l k z}+n 7_{i t l k z}+n 8_{i t l k z}\right.$ $\left.+n 9_{i t l k z}+n 10_{i t l k z}+n 11_{i t l k z}\right) M$,

$$
\begin{aligned}
e w_{i t l k z}^{\prime} \leq(110 & \left.\times y 4_{i t l k z}\right)+\left(1-n 4_{i t l k z}\right) M \\
& +\left(n 1_{i t l k z}+n 2_{i t l k z}+n 3_{i t l k z}+n 5_{i t l k z}+n 6_{i t l k z}+n 7_{i t l k z}+n 8_{i t l k z}\right. \\
& \left.+n 9_{i t l k z}+n 10_{i t l k z}+n 11_{i t l k z}\right) M,
\end{aligned}
$$

$e w_{i t l k z}^{\prime} \geq\left(71 \times y 4_{i t l k z}\right)-\left(1-n 4_{i t l k z}\right) M$

$$
\begin{aligned}
& -\left(n 1_{i t l k z}+n 2_{i t l k z}+n 3_{i t l k z}+n 5_{i t l k z}+n 6_{i t l k z}+n 7_{i t l k z}+n 8_{i t l k z}\right. \\
& \left.+n 9_{i t l k z}+n 10_{i t l k z}+n 11_{i t l k z}\right) M,
\end{aligned}
$$

$e w_{i t l k z}^{\prime} \leq\left(133 \times y 5_{i t l k z}\right)+\left(1-n 5_{i t l k z}\right) M$

$$
+\left(n 1_{i t l k z}+n 2_{i t l k z}+n 3_{i t l k z}+n 4_{i t l k z}+n 6_{i t l k z}+n 7_{i t l k z}+n 8_{i t l k z}\right.
$$$$
\left.+n 9_{i t l k z}+n 10_{i t l k z}+n 11_{i t l k z}\right) M \text {, }
$$

$e w_{i t l k z}^{\prime} \geq\left(111 \times y 5_{i t l k z}\right)-\left(1-n 5_{i t l k z}\right) M$

$-\left(n 1_{i t l k z}+n 2_{i t l k z}+n 3_{i t l k z}+n 4_{i t l k z}+n 6_{i t l k z}+n 7_{i t l k z}+n 8_{i t l k z}\right.$ $\left.+n 9_{i t l k z}+n 10_{i t l k z}+n 11_{i t l k z}\right) M$,

$e w_{i t l k z}^{\prime} \leq\left(157 \times y 6_{i t l k z}\right)+\left(1-n 6_{i t l k z}\right) M$

$+\left(n 1_{i t l k z}+n 2_{i t l k z}+n 3_{i t l k z}+n 4_{i t l k z}+n 5_{i t l k z}+n 7_{i t l k z}+n 8_{i t l k z}\right.$ $\left.+n 9_{i t l k z}+n 10_{i t l k z}+n 11_{i t l k z}\right) M$,

$e w_{i t l k z}^{\prime} \geq\left(134 \times y 6_{i t l k z}\right)-\left(1-n 6_{i t l k z}\right) M$

$-\left(n 1_{i t l k z}+n 2_{i t l k z}+n 3_{i t l k z}+n 4_{i t l k z}+n 5_{i t l k z}+n 7_{i t l k z}+n 8_{i t l k z}\right.$ $\left.+n 9_{i t l k z}+n 10_{i t l k z}+n 11_{i t l k z}\right) M$,

$e w_{i t l k z}^{\prime} \leq\left(195 \times y 7_{i t l k z}\right)+\left(1-n 7_{i t l k z}\right) M$

$+\left(n 1_{i t l k z}+n 2_{i t l k z}+n 3_{i t l k z}+n 4_{i t l k z}+n 5_{i t l k z}+n 6_{i t l k z}+n 8_{i t l k z}\right.$ $\left.+n 9_{i t l k z}+n 10_{i t l k z}+n 11_{i t l k z}\right) M$,

$e w_{i t l k z}^{\prime} \geq\left(158 \times y 7_{i t l k z}\right)-\left(1-n 7_{i t l k z}\right) M$

$-\left(n 1_{i t l k z}+n 2_{i t l k z}+n 3_{i t l k z}+n 4_{i t l k z}+n 5_{i t l k z}+n 6_{i t l k z}+n 8_{i t l k z}\right.$

$e w_{i t l k z}^{\prime} \leq\left(233 \times y 8_{i t l k z}\right)+\left(1-n 8_{i t l k z}\right) M$

$+\left(n 1_{i t l k z}+n 2_{i t l k z}+n 3_{i t l k z}+n 4_{i t l k z}+n 5_{i t l k z}+n 6_{i t l k z}+n 7_{i t l k z}\right.$

$e w_{i t l k z}^{\prime} \geq\left(196 \times y 8_{i t l k z}\right)-\left(1-n 8_{i t l k z}\right) M$

$-\left(n 1_{i t l k z}+n 2_{i t l k z}+n 3_{i t l k z}+n 4_{i t l k z}+n 5_{i t l k z}+n 6_{i t l k z}+n 7_{i t l k z}\right.$

$e w_{i t l k z}^{\prime} \leq\left(265 \times y 9_{i t l k z}\right)+\left(1-n 9_{i t l k z}\right) M$

$+\left(n 1_{i t l k z}+n 2_{i t l k z}+n 3_{i t l k z}+n 4_{i t l k z}+n 5_{i t l k z}+n 6_{i t l k z}+n 7_{i t l k z}\right.$

$e w_{i t l k z}^{\prime} \geq\left(234 \times y 9_{i t l k z}\right)-\left(1-n 9_{i t l k z}\right) M$

$-\left(n 1_{i t l k z}+n 2_{i t l k z}+n 3_{i t l k z}+n 4_{i t l k z}+n 5_{i t l k z}+n 6_{i t l k z}+n 7_{i t l k z}\right.$

$e w_{i t l k z}^{\prime} \leq\left(297 \times y 10_{i t l k z}\right)+\left(1-n 10_{i t l k z}\right) M$

$+\left(n 1_{i t l k z}+n 2_{i t l k z}+n 3_{i t l k z}+n 4_{i t l k z}+n 5_{i t l k z}+n 6_{i t l k z}+n 7_{i t l k z}\right.$

$e w_{i t l k z}^{\prime} \geq\left(266 \times y 10_{i t l k z}\right)-\left(1-n 10_{i t l k z}\right) M$

$$
-\left(n 1_{i t l k z}+n 2_{i t l k z}+n 3_{i t l k z}+n 4_{i t l k z}+n 5_{i t l k z}+n 6_{i t l k z}+n 7_{i t l k z}\right.
$$

$$
\left.+n 8_{i t l k z}+n 9_{i t l k z}+n 11_{i t l k z}\right) M \text {, }
$$




$$
\begin{aligned}
e w_{i t l k z}^{\prime} \leq(334 & \left.\times y 11_{i t l k z}\right)+\left(1-n 11_{i t l k z}\right) M \\
& +\left(n 1_{i t l k z}+n 2_{i t l k z}+n 3_{i t l k z}+n 4_{i t l k z}+n 5_{i t l k z}+n 6_{i t l k z}+n 7_{i t l k z}\right. \\
& \left.+n 8_{i t l k z}+n 9_{i t l k z}+n 10_{i t l k z}\right) M \\
e w_{i t l k z}^{\prime} \geq(298 & \left.\times y 11_{i t l k z}\right)-\left(1-n 11_{i t l k z}\right) M \\
& -\left(n 1_{i t l k z}+n 2_{i t l k z}+n 3_{i t l k z}+n 4_{i t l k z}+n 5_{i t l k z}+n 6_{i t l k z}+n 7_{i t l k z}\right. \\
& \left.+n 8_{i t l k z}+n 9_{i t l k z}+n 10_{i t l k z}\right) M, \\
n 1_{i t l k z}+n 2_{i t l k z} & +n 3_{i t l k z}+n 4_{i t l k z}+n 5_{i t l k z}+n 6_{i t l k z}+n 7_{i t l k z}+n 8_{i t l k z}+n 9_{i t l k z} \\
& +n 10_{i t l k z}+n 11_{i t l k z}=y_{i t l k z}, \forall i \in I, \forall t \in T, \forall l \in L, k \in K, z \in Z .
\end{aligned}
$$

The amount of electrical current between two consumers is given by constraints (26)-(29). Amount of crowded electrical current in a consumer for supporting other consumers is shown by constraint (30). Constraints (31)-(52) represent different amounts of electric currents between two consumers corresponding to Table 1. Constraint (53) guarantees that amount of electrical current flow between two consumers can accept just one available mood of Table 1. Constraints (54)-(57) specify the amount of electrical current between transformers and consumers. Different amounts of electrical current between transformer and consumer are presented by constraints (58)-(79) according to Table 1. Constraint (80) certifies that amount of electric current between transformer and consumer can choose exactly one valid situation as given in Table 1.

\subsubsection{Capacity Constraint}

$$
\sum_{z \in Z} e w_{i t l k z}^{\prime} \leq Q 3_{i t l k}, \quad \forall i \in I, \forall t \in T, \forall l \in L, k \in K, z \in Z
$$

\subsubsection{Feeder Length Constraints}

$$
\begin{gathered}
M\left(w_{z z^{\prime}}-1\right)+\left(d_{z z^{\prime}}^{\prime \prime}+d w_{z^{\prime}}\right) \leq f d_{z z^{\prime}}, \forall z, z^{\prime} \in Z, \\
\left(w_{z z^{\prime}}-1\right)(-M)+\left(d_{z z^{\prime}}^{\prime \prime}+d w_{z^{\prime}}\right) \geq f d_{z z^{\prime}}, \forall z, z^{\prime} \in Z, \\
f d_{z z^{\prime}} \leq w_{z z^{\prime}} M, \quad \forall z, z^{\prime} \in Z, \\
f d_{z z^{\prime}} \geq w_{z z^{\prime}}, \quad \forall z, z^{\prime} \in Z, \\
\sum_{z \prime \in Z} f d_{z z^{\prime}}=d w_{z}, \quad \forall z \in Z, \\
\left(y_{i t l k z}-1\right) M+\left(d_{i z}+d w_{z}\right) \leq d w_{i t l k z}^{\prime}, \quad \forall i \in I, \forall t \in T, \forall l \in L, k \in K, z \in Z,
\end{gathered}
$$




$$
\begin{gathered}
\left(y_{i t l k z}-1\right)(-M)+\left(d_{i z}+d w_{z}\right) \geq d w_{i t l k z}^{\prime}, \quad \forall i \in I, \forall t \in T, \forall l \in L, \forall k \in K, \forall z \in Z, \\
d w_{i t l k z}^{\prime} \leq M y_{i t l k z}, \forall i \in I, \forall t \in T, \forall l \in L, k \in K, z \in Z, \\
d w_{i t l k z}^{\prime} \geq y_{i t l k z}, \forall i \in I, \forall t \in T, \forall l \in L, k \in K, z \in Z, \\
\sum_{z \in Z} d w_{i t l k z}^{\prime} \leq 300, \forall i \in I, \forall t \in T, \forall l \in L, k \in K, z \in Z .
\end{gathered}
$$

Constraints (82)-(91) ensures that the length of each feeder is less than 300 meters.

\subsubsection{Constraints on Variables}

$$
\begin{gathered}
r_{i}, h_{i t}, a_{i t l}, v_{i t l k}, y_{i t l k z}, u_{i}, x_{i i^{\prime}}, w_{z z^{\prime}} \in\{0,1\}, \forall i, i^{\prime} \in I, \forall t \in T, \forall l \in L, \forall k \in K, \forall z, z^{\prime} \in Z, \\
y 1_{i t l k z}, \ldots, y 11_{i t l k z} \in\{0,1\}, \quad \forall i, i^{\prime} \in I, \forall t \in T, \forall l \in L, \forall k \in K, \forall z, z^{\prime} \in Z, \\
n 1_{i t l k z}, \ldots, n 11_{i t l k z} \in\{0,1\}, \quad \forall i \in I, \forall t \in T, \forall l \in L, \forall k \in K, \forall z \in Z \\
\qquad \begin{array}{c}
w 1_{z z^{\prime}}, \ldots, w 11_{z z^{\prime}} \in\{0,1\}, \forall z, z^{\prime} \in Z \\
N_{z}, f_{i i^{\prime}}, f_{z z^{\prime}}^{\prime}, f_{z z^{\prime}}^{\prime \prime}, f d_{z z^{\prime}}, e w_{z}, e w_{i t l k z}^{\prime}, d w_{z}, d w_{i t l k z}^{\prime} \geq 0 \\
\forall i, i^{\prime} \in I, \forall t \in T, \forall l \in L, \forall k \in K, \forall z, z^{\prime} \in Z
\end{array}
\end{gathered}
$$

Constraint (92) shows the binary variables and constraint (93) specifies the nonnegative variable.

\section{Proposed Genetic Algorithm}

Our proposed model is based on the minimum spanning tree (MST) method which is classified as an NP-hard problem. Because of this, exact methods need excessive computational time. So, to solve these problems in reasonable amounts of time, utilizing heuristic and meta-heuristic algorithm is necessary. However, such algorithms do not necessarily find global optimal solutions, but yield good solutions in reasonable times. According to our proposed mathematical model, our problem belongs to the discrete optimization problems.

The genetic algorithm (GA) has rich applications to discrete optimization problems (e.g., TSP, MST, Vehicle Routing, and Network Routing problems) and converges to good quality solutions rapidly. The proposed GA algorithm also solves large size problems. The GA is expected to obtain 
high-quality solutions and is appropriate for solving problems with specifications such as abstruseness of computations and limited memory of computers.

Genetic algorithms are based on some biological operators like selection, crossover, and mutation. A population of candidate solutions in the genetic algorithm is called individuals each having some specifications called chromosome that can be changed to a better situation. Individuals are submitted as an array of bits and usually are shown by binary strings.

There are terms such as generation and fitness value in a genetic algorithm. Generation is the evolution of the population in each repetition, and each generation of candidate solutions is used in the next iteration. The fitness value is defined as a value of the objective function evaluated in each generation. Termination of genetic algorithm usually depends on two conditions, one is number of generations, and another is the fitness level of the population.

\subsection{Initialization}

According to the specifications of our problem, initial population size can consist of several possible solutions. Selection of initial population is made from the whole space of search area.

\subsection{Selection}

A new generation is created by choosing some members of the existing population. Choosing of individual solutions is done by a process based on the fitness function. Consequently, quality of submitted solutions is measured by a fitness function which relies on the problem, meaning that solutions with better fitness value are selected.

\subsection{GA Operators}

Our next step is generating a second generation of individuals from those selected through a mixture of crossover and mutation operators. The role of crossover operator is selecting a collection of good chromosomes for procreation, and it means this operator combines chromosomes of parents and generates new chromosomes.

Mutation operator is used for recovery of missed information and is a useful tool for rapid convergence and also helps the search process to escape from local optima. By comparing these two operators, we understand that crossover operation just combines the information from parents, while the mutation operator adds some new information to the problem.

\subsection{Termination Condition}

The stopping condition may be provided in various ways. Here, our stopping condition for our proposed GA algorithm is reaching a maximum number of allowable repetitions in the algorithm.

\subsection{Algorithm}

The main steps of our proposed GA algorithm is shown in Fig. 3. 


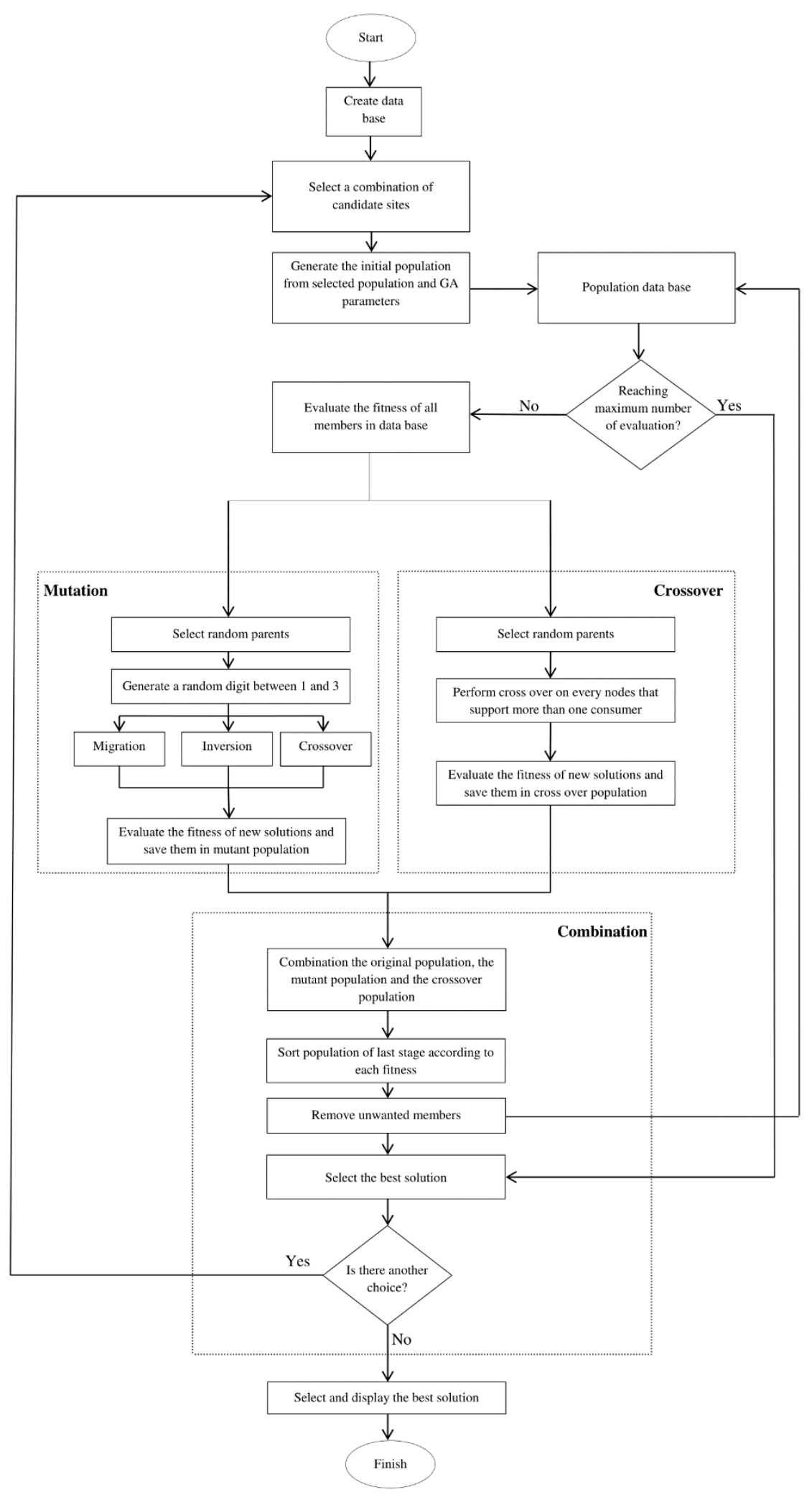

Figure 3. Steps of the proposed GA algorithm 


\section{A Case Study}

A case study of power network in Tehran Power Distribution Company in Iran is made to validate the proposed model. By survey on our case study, we considered four potential locations of transformers supporting 15 consumption zones with distinct single-phase demands. Table 1 shows the relationships between electric current rates and low voltage cables sizes and the costs of low voltage cabling per meter. Consumers' demands are presented in Table 2. There are six kinds of transformers with various capacities in the network as given in Table 3. To find a spanning tree with minimum distance of links, the MST method was applied.

We have three kinds of connection links between transformers and consumers, two transformers, and two consumers.

The number of each kind of transformer feeder and capacity of each feeder is shown in Table 4.

Table 1. The cost of low voltage cabling per unit distance according to current rate.

\begin{tabular}{|c|c|c|c|}
\hline Electrical Current Rate (Ampere) & $\begin{array}{c}\text { Cable } \\
\text { Size(mm2) }\end{array}$ & $\begin{array}{c}\text { Cables } \\
\text { Type }\end{array}$ & $\begin{array}{c}\text { Cost } \\
\text { (Rials) }\end{array}$ \\
\hline$[0-30)$ & 6 & $\mathrm{cu}$ & 108000 \\
\hline$[30-45)$ & 10 & $\mathrm{cu}$ & 163000 \\
\hline$[45-70)$ & 16 & $\mathrm{cu}$ & 195000 \\
\hline$[70-110)$ & 25 & $\mathrm{cu}$ & 276500 \\
\hline$[110-133)$ & 35 & $\mathrm{cu}$ & 354800 \\
\hline$[133-157)$ & 50 & $\mathrm{cu}$ & 464000 \\
\hline$[157-195)$ & 70 & $\mathrm{cu}$ & 627900 \\
\hline$[195-233)$ & 95 & $\mathrm{cu}$ & 839900 \\
\hline$[233-265)$ & 120 & $\mathrm{cu}$ & 1120600 \\
\hline$[265-297)$ & 150 & $\mathrm{cu}$ & 1287100 \\
\hline$[297-334]$ & 180 & $\mathrm{cu}$ & 1647100 \\
\hline
\end{tabular}

Table 2. Consumer's single phase demand in Ampere.

\begin{tabular}{|c|c|c|c|c|c|c|c|c|c|c|c|c|c|c|c|}
\hline Consumer & $\mathbf{1}$ & $\mathbf{2}$ & $\mathbf{3}$ & $\mathbf{4}$ & $\mathbf{5}$ & $\mathbf{6}$ & $\mathbf{7}$ & $\mathbf{8}$ & $\mathbf{9}$ & $\mathbf{1 0}$ & $\mathbf{1 1}$ & $\mathbf{1 2}$ & $\mathbf{1 3}$ & $\mathbf{1 4}$ & $\mathbf{1 5}$ \\
\hline $\begin{array}{c}\text { Demand } \\
\text { (Ampere) }\end{array}$ & 50 & 32 & 25 & 100 & 96 & 32 & 50 & 15 & 25 & 32 & 50 & 32 & 96 & 45 & 50 \\
\hline
\end{tabular}


Table 3. Types, costs, and capacities of transformers.

\begin{tabular}{|c|c|c|c|}
\hline Transformer & Capacity (KVA) & Each phase capacity (Ampere) & Cost (Rials) \\
\hline $\mathbf{1}$ & 50 & 72 & 90997000 \\
\hline $\mathbf{2}$ & 100 & 144 & 134742000 \\
\hline $\mathbf{3}$ & 200 & 288 & 198860000 \\
\hline $\mathbf{4}$ & 250 & 360 & 228000000 \\
\hline $\mathbf{5}$ & 315 & 454 & 269240000 \\
\hline $\mathbf{6}$ & 400 & 576 & 329160000 \\
\hline
\end{tabular}

Table 4. Capacity of each phase of transformer feeder.

\begin{tabular}{|c|c|c|c|c|c|}
\hline \multirow{2}{*}{$\begin{array}{c}\text { Transformer } \\
\text { Type }\end{array}$} & Number of Feeders & \multirow{2}{*}{ Feeder No. } & \multicolumn{2}{|c|}{ Each phase capacity (Ampere) } \\
\cline { 3 - 5 } & & & $\mathrm{R}$ & $\mathrm{S}$ & $\mathrm{T}$ \\
\hline $\mathbf{1}$ & 1 & 1 & 72 & 72 & 72 \\
\hline $\mathbf{2}$ & 1 & 1 & 144 & 144 & 144 \\
\hline $\mathbf{3}$ & 1 & 1 & 288 & 288 & 288 \\
\hline \multirow{2}{*}{$\mathbf{4}$} & \multirow{2}{*}{2} & 1 & 300 & 300 & 300 \\
\hline \multirow{2}{*}{$\mathbf{5}$} & \multirow{2}{*}{2} & 2 & 160 & 160 & 160 \\
\hline \multirow{2}{*}{$\mathbf{6}$} & \multirow{2}{*}{2} & 2 & 250 & 250 & 250 \\
\hline & & 1 & 204 & 204 & 204 \\
\hline & & 2 & 315 & 315 & 315 \\
\hline
\end{tabular}

The corresponding distances with connection links are shown in Tables 5-7. The average cost of medium voltage cabling per distance unit between the transformers is determined to be 297300 Rials. Disposition cost of transformers and capacity of various types of transformers is indicated in Table 3.

Table 5. Distance among the sites and consumers.

\begin{tabular}{|c|c|c|c|c|c|c|c|c|}
\hline $\begin{array}{c}\text { Sites/ } \\
\text { Consumers }\end{array}$ & $\mathbf{1}$ & $\mathbf{2}$ & $\mathbf{3}$ & $\mathbf{4}$ & $\mathbf{5}$ & $\mathbf{6}$ & $\mathbf{7}$ & $\mathbf{8}$ \\
\hline $\mathbf{1}$ & 164.5 & 153 & 134.6 & 126 & 116 & 95 & 86.4 & 74.6 \\
\hline $\mathbf{2}$ & 312 & 296 & 286 & 276.2 & 257.3 & 246.7 & 237.4 & 228.13 \\
\hline $\mathbf{3}$ & 429.1 & 413.4 & 403.2 & 392.4 & 373.5 & 364.6 & 354 & 343.9 \\
\hline $\mathbf{4}$ & 619.8 & 604.4 & 594.1 & 584.5 & 564.5 & 554.9 & 545.2 & 534.5 \\
\hline
\end{tabular}


Table 5. Distance among the sites and consumers (continued).

\begin{tabular}{|c|c|c|c|c|c|c|c|}
\hline $\begin{array}{c}\text { Sites/ } \\
\text { Consumers }\end{array}$ & $\mathbf{9}$ & $\mathbf{1 0}$ & $\mathbf{1 1}$ & $\mathbf{1 2}$ & $\mathbf{1 3}$ & $\mathbf{1 4}$ & $\mathbf{1 5}$ \\
\hline $\mathbf{1}$ & 64 & 56 & 45 & 36 & 26 & 16 & 6 \\
\hline $\mathbf{2}$ & 217.3 & 208.2 & 197 & 186.2 & 177.2 & 166.8 & 157.5 \\
\hline $\mathbf{3}$ & 333.8 & 320.16 & 313.34 & 302.5 & 293.4 & 283.3 & 273.5 \\
\hline $\mathbf{4}$ & 524.4 & 515.7 & 503.8 & 494.2 & 484.1 & 474.3 & 465.8 \\
\hline
\end{tabular}

Table 6. Distance among the sites.

\begin{tabular}{|c|c|c|c|c|}
\hline Sites & $\mathbf{1}$ & $\mathbf{2}$ & $\mathbf{3}$ & $\mathbf{4}$ \\
\hline $\mathbf{1}$ & 0 & 171 & 296 & 500 \\
\hline $\mathbf{2}$ & 171 & 0 & 125 & 329 \\
\hline $\mathbf{3}$ & 296 & 125 & 0 & 204 \\
\hline $\mathbf{4}$ & 500 & 329 & 204 & 0 \\
\hline
\end{tabular}

Table 7. Distance among consumers.

\begin{tabular}{|c|c|c|c|c|c|c|c|c|}
\hline Consumers & $\mathbf{1}$ & $\mathbf{2}$ & $\mathbf{3}$ & $\mathbf{4}$ & $\mathbf{5}$ & $\mathbf{6}$ & $\mathbf{7}$ & $\mathbf{8}$ \\
\hline $\mathbf{1}$ & 0 & 20.48 & 36.0 & 46.23 & 56.33 & 75.84 & 85.94 & 95.99 \\
\hline $\mathbf{2}$ & 20.48 & 0 & 15.57 & 25.57 & 35.84 & 55.37 & 65.64 & 75.52 \\
\hline $\mathbf{3}$ & 36.05 & 15.57 & 0 & 10.18 & 20.28 & 39.8 & 49.89 & 59.95 \\
\hline $\mathbf{4}$ & 46.23 & 25.75 & 10.18 & 0 & 10.09 & 29.62 & 39.72 & 49.77 \\
\hline $\mathbf{5}$ & 56.33 & 35.84 & 20.28 & 10.09 & 0 & 19.52 & 29.62 & 39.67 \\
\hline $\mathbf{6}$ & 75.84 & 55.37 & 39.8 & 29.62 & 19.52 & 0 & 10.09 & 20.15 \\
\hline $\mathbf{7}$ & 85.94 & 65.46 & 49.89 & 39.72 & 29.62 & 10.09 & 0 & 10.05 \\
\hline $\mathbf{8}$ & 95.99 & 75.52 & 59.95 & 49.77 & 39.67 & 20.15 & 10.05 & 0 \\
\hline $\mathbf{9}$ & 105.83 & 85.36 & 69.78 & 59.61 & 49.51 & 29.98 & 19.89 & 9.84 \\
\hline $\mathbf{1 0}$ & 116.05 & 95.57 & 80 & 69.82 & 59.73 & 40.02 & 30.10 & 20.05 \\
\hline $\mathbf{1 1}$ & 126.01 & 105.53 & 89.96 & 79.78 & 69.68 & 50.16 & 40.07 & 30.02 \\
\hline $\mathbf{1 2}$ & 135.96 & 115.48 & 99.9 & 89.7 & 79.63 & 60.11 & 50.01 & 39.96 \\
\hline $\mathbf{1 3}$ & 146.03 & 125.55 & 109.98 & 99.8 & 89.71 & 70.18 & 60.09 & 50.04 \\
\hline $\mathbf{1 4}$ & 156.01 & 135.53 & 119.96 & 109.78 & 99.68 & 80.16 & 70.1 & 60.02 \\
\hline $\mathbf{1 5}$ & 166.06 & 145.59 & 130.02 & 119.84 & 109.74 & 90.22 & 80.13 & 70.07 \\
\hline
\end{tabular}


Table 7. Distance among consumers (continued).

\begin{tabular}{|c|c|c|c|c|c|c|c|}
\hline Consumers & $\mathbf{9}$ & $\mathbf{1 0}$ & $\mathbf{1 1}$ & $\mathbf{1 2}$ & $\mathbf{1 3}$ & $\mathbf{1 4}$ & $\mathbf{1 5}$ \\
\hline $\mathbf{1}$ & 105.83 & 116.05 & 126.01 & 135.96 & 146.03 & 156.01 & 166.06 \\
\hline $\mathbf{2}$ & 85.36 & 95.57 & 105.53 & 115.48 & 125.55 & 135.53 & 145.59 \\
\hline $\mathbf{3}$ & 69.78 & 80 & 89.96 & 99.9 & 109.98 & 119.96 & 130.02 \\
\hline $\mathbf{4}$ & 59.61 & 69.82 & 79.78 & 89.7 & 99.8 & 109.78 & 119.84 \\
\hline $\mathbf{5}$ & 49.51 & 59.73 & 69.68 & 79.63 & 89.71 & 99.68 & 109.74 \\
\hline $\mathbf{6}$ & 29.98 & 40.02 & 50.16 & 60.11 & 70.18 & 80.16 & 90.22 \\
\hline $\mathbf{7}$ & 19.89 & 30.10 & 40.07 & 50.01 & 60.09 & 70.10 & 80.13 \\
\hline $\mathbf{8}$ & 9.84 & 20.05 & 30.02 & 39.96 & 50.04 & 60.02 & 70.07 \\
\hline $\mathbf{9}$ & 0 & 10.21 & 20.18 & 30.12 & 40.19 & 50.17 & 60.23 \\
\hline $\mathbf{1 0}$ & 10.021 & 0 & 9.96 & 19.91 & 29.98 & 39.96 & 50.02 \\
\hline $\mathbf{1 1}$ & 20.18 & 9.96 & 0 & 9.94 & 20.02 & 29.99 & 40.06 \\
\hline $\mathbf{1 2}$ & 30.12 & 19.91 & 9.94 & 0 & 10.07 & 20.05 & 30.11 \\
\hline $\mathbf{1 3}$ & 40.19 & 29.98 & 20.02 & 10.07 & 0 & 9.98 & 20.04 \\
\hline $\mathbf{1 4}$ & 50.17 & 39.96 & 29.99 & 20.05 & 9.98 & 0 & 20.14 \\
\hline $\mathbf{1 5}$ & 60.23 & 50.02 & 40.06 & 30.11 & 20.04 & 20.14 & 0 \\
\hline
\end{tabular}

We applied GAMS 24.7.4 software package for solving our proposed Mixed Integer Programming (MIP) model. GAMS software is appropriate for linear optimization problems, and, in particular, quadratic programs [20]. The correctness of our proposed model is assessed for our case study. According to the large-scale and long solving time of the problem, first we minimize the scale of the problem assuming 4 sites, 2 kinds of transformer with capacities of 315 and $400 \mathrm{KVA}$, 2 feeders for each transformer and minimized demands of consumers as shown in Table 8. The results are presented in Table 9.

Note that we have two kinds of connection links in the selected path column:

- Joined links between the located transformer in site $i$ and consumer $z$ which is presented by [a-b-c-d): [e] format, where $a, b, c$, and $d$ respectively express the number of selected transformer, feeder, phase and consumer, and $e$ is a number which indicates a selected site, and [ ): [ ] shows the type of connection links.

- (f-g) format expressing links connected between the consumers, where, $f$ and $g$ respectively give number of selected consumers, and () is the corresponding sign to the type of connection links.

Table 8. Consumer's single phase minimized demand in Ampere.

\begin{tabular}{|c|c|c|c|c|c|c|c|c|c|}
\hline Consumer & $\mathbf{1}$ & $\mathbf{2}$ & $\mathbf{3}$ & $\mathbf{4}$ & $\mathbf{5}$ & $\mathbf{6}$ & $\mathbf{7}$ & $\mathbf{8}$ & $\mathbf{9}$ \\
\hline $\begin{array}{c}\text { Demand } \\
\text { (Ampere) }\end{array}$ & 17.5 & 22.4 & 53 & 42 & 30 & 53 & 88 & 14 & 11.2 \\
\hline
\end{tabular}


Table 9. Result of solving the minimized scale problem with GAMS.

\begin{tabular}{|c|c|c|}
\hline $\begin{array}{l}\text { Selected } \\
\text { path }\end{array}$ & $\begin{array}{l}\text { Amount of electrical } \\
\text { current (Ampere) }\end{array}$ & $\begin{array}{c}\text { Cable } \\
\text { Size } \\
(\mathrm{mm} 2) \\
\end{array}$ \\
\hline [5-1-1-9): [1] & 106.2 & 25 \\
\hline$(9-6)$ & 95 & 25 \\
\hline$(6-4)$ & 42 & 10 \\
\hline [5-1-2-8): [1] & 102 & 25 \\
\hline$(8-7)$ & 88 & 25 \\
\hline [5-1-3-5): [1] & 122.9 & 35 \\
\hline$(5-3)$ & 92.9 & 25 \\
\hline$(3-2)$ & 39.9 & 10 \\
\hline$(2-1)$ & 17.5 & 6 \\
\hline \multicolumn{3}{|c|}{ Optimal objective value } \\
\hline \multicolumn{3}{|c|}{373590000 (Rials) } \\
\hline \multicolumn{3}{|c|}{ Elapsed time } \\
\hline \multicolumn{3}{|c|}{$595775.44 \mathrm{sec}$} \\
\hline
\end{tabular}

The optimal objective function for the minimized problem is 373590000 Rials obtained in 595775.44 seconds. Note that this computing time was spent on solving a problem with four potential locations for the transformers and nine consumer zones. Since the real case at Tehran Power Distribution Company contains 15 consumption zones with four possible locations for the transformers, GAMS is unable to attain solutions for 15 consumer zones in an acceptable time. Due to the complexity of these problems, exact methods need excessive computing times. So, the GA algorithm is an essential tool for solving such problems in reasonable amounts of time. However, such algorithms do not necessarily always find global optimal solutions, but yield good solutions in reasonable times. The test problems are produced to present the validity and efficiency of our proposed method. These problems are based on four factors of number of consumers, feeders, sites, and transformers.

Since the proposed model was formulated for the case study of Tehran Power Distribution Company, the generation of test problems is based on real data. Due to the limited capability of GAMS 24.7.4 software package for solving our problems in reasonable times, the test problems were produced in sizes convenient for the software to solve. The test problems are given as follows:

- Four consumers, one site, two transformers and two feeders

- Four consumers, two sites, two transformers and two feeders

- Five consumers, one site, two transformers and two feeders

- Five consumers, two sites, two transformers and two feeders

- Six consumers, one site, two transformers and two feeders

- Six consumers, two sites, two transformers and two feeders

- Six consumers, three sites, two transformers and two feeders

- Six consumers, four sites, two transformers and two feeders 
- Seven consumers, four sites, two transformers and two feeders

- Nine consumers, four sites, two transformers and two feeders

Different values for parameters of the algorithm influence the desirability of obtained solutions. So, there are solutions with different qualities based on various combinations of parameters. Parameter tuning plays a vital role in the algorithm to produce desirable solutions. First, we introduce several levels for each parameter. A different combination of parameter's levels is defined as a test plan. Then, the test plans are implemented to determine a suitable level for each parameter.

The levels of parameters are given as follows:

- The number of population (pop): three levels $(40,50,60)$.

- Crossover percentage (pc): three levels $(0.7,0.8,0.9)$.

- Mutation percentage (pm): three levels $(0.1,0.2,0.3)$.

- Selection pressure (sp): three levels $(5,10,15)$.

There are $3 * 3 * 3 * 3=81$ test plans. Each test plan is implemented for each defined test problem, and the obtained results are saved using the following relation:

$$
\text { Gap }=\frac{\left|f_{G A}-f_{\text {optimal }}\right|}{f_{\text {optimal }}} \times 100
$$

where $f_{G A}$ is a fitness function of the proposed GA algorithm and $f_{\text {optimal }}$ is an optimal solution obtained by employing the exact method. The test problem's gap is considered to compute the mean of the gap for each test plan. The Minitab 17 software package was applied to examine the effect of various parameters on the value of the gap. The results are shown in Fig. 4. Corresponding to the obtained results, levels 40, 0.9, 0.1 and 15 were selected for the number of population (pop), Crossover percentage (pc), Mutation percentage (pm), and Selection pressure (sp), respectively.

\subsection{Computational Results}

Here, the validity and productivity of the proposed GA algorithm in comparison to the exact method is analyzed. The computational test was developed on a personal computer with Intel (R) core i7 with $3.1 \mathrm{GHz}$ CPU / 16 GB RAM. The algorithm was coded using MATLAB R2016 software package. We generated 10 test problems to test our approach. The results are given in Table 10. The results show that our GA algorithm is effective to solve the problems. The algorithm obtains good solutions in reasonable times.

In comparison to the exact method, the algorithm obtains solutions closer to the optimal solutions with much less time than the time needed to get the exact optimal solution. The applied GA approach in the real case at Tehran Power Distribution Company provided 2915069092 Rials of cost.

After the accrediting validity of the proposed GA algorithm, we applied it to the main problem in large scale and achieved results as shown in tables 11 and 12. Fig. 5 shows how the consumers are allocated to the different phases of two transformers. 

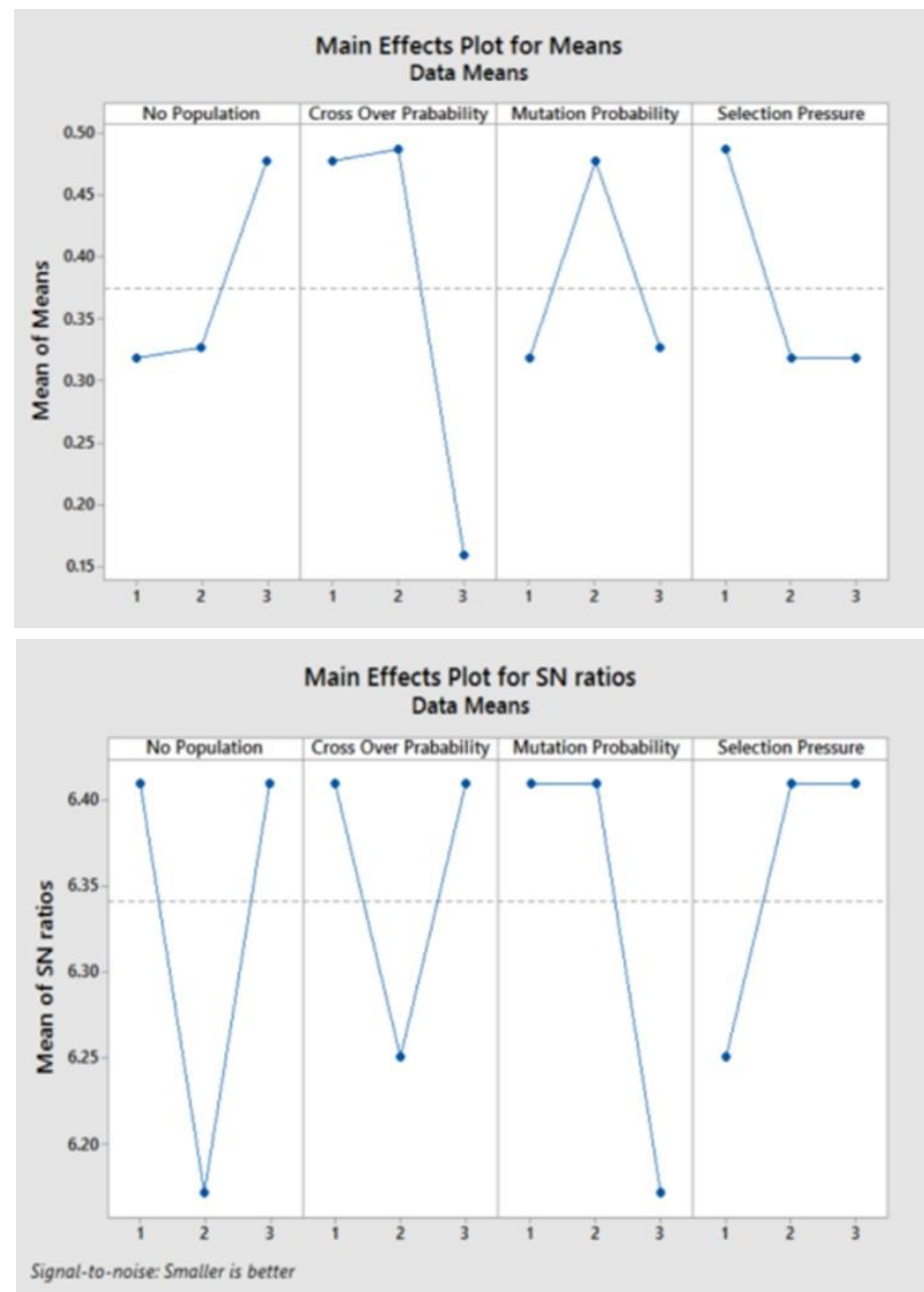

Figure 4. Impact of each parameter on mean of gap 
Table 10. Computational results.

\begin{tabular}{|c|c|c|c|c|c|c|}
\hline \multirow{3}{*}{ Num } & \multirow{3}{*}{$\begin{array}{l}\text { Site } \\
\text { No. }\end{array}$} & \multirow{3}{*}{$\begin{array}{c}\text { No. of } \\
\text { Consumers }\end{array}$} & \multirow{3}{*}{$\begin{array}{l}\text { No. of } \\
\text { Feeders }\end{array}$} & $\begin{array}{c}\text { GA } \\
\text { Algorithm }\end{array}$ & GAMS 27.7.4 & \multirow{3}{*}{$\begin{array}{c}\text { Gap } \\
(\%)\end{array}$} \\
\hline & & & & Response & Response & \\
\hline & & & & Time & Time & \\
\hline \multirow{2}{*}{1} & \multirow{2}{*}{1} & \multirow{2}{*}{4} & \multirow{2}{*}{2} & 334246134 & 334246100 & \multirow{2}{*}{$1.01 \mathrm{E}-07$} \\
\hline & & & & $16.62 \mathrm{sec}$ & $2.359 \mathrm{sec}$ & \\
\hline \multirow{2}{*}{2} & \multirow{2}{*}{2} & \multirow{2}{*}{4} & \multirow{2}{*}{2} & 334246134 & 334246100 & \multirow{2}{*}{$1.01 \mathrm{E}-07$} \\
\hline & & & & $17.398 \mathrm{sec}$ & $4.25 \mathrm{sec}$ & \\
\hline \multirow{2}{*}{3} & \multirow{2}{*}{1} & \multirow{2}{*}{5} & \multirow{2}{*}{2} & 339862866 & 338525600 & \multirow{2}{*}{ 3.95E-03 } \\
\hline & & & & $16.176 \mathrm{sec}$ & $26.094 \mathrm{sec}$ & \\
\hline \multirow{2}{*}{4} & \multirow{2}{*}{2} & \multirow{2}{*}{5} & \multirow{2}{*}{2} & 339862866 & 338525600 & \multirow{2}{*}{$3.95 \mathrm{E}-03$} \\
\hline & & & & $12.296 \mathrm{sec}$ & $47.75 \mathrm{sec}$ & \\
\hline \multirow{2}{*}{5} & \multirow{2}{*}{1} & \multirow{2}{*}{6} & \multirow{2}{*}{2} & 353601534 & 351174600 & \multirow{2}{*}{$6.9 \mathrm{E}-03$} \\
\hline & & & & $17.162 \mathrm{sec}$ & $184.547 \mathrm{sec}$ & \\
\hline \multirow{2}{*}{6} & \multirow{2}{*}{2} & \multirow{2}{*}{6} & \multirow{2}{*}{2} & 353601534 & 351174600 & 6 OF_03 \\
\hline & & & & $17.534 \mathrm{sec}$ & $221.422 \mathrm{sec}$ & $0.9 \mathrm{E}-03$ \\
\hline 7 & 3 & 6 & 2 & 353601534 & 351174600 & $60 \mathrm{~F}_{0} 03$ \\
\hline 1 & 3 & 0 & 2 & $17.782 \mathrm{sec}$ & $78.969 \mathrm{sec}$ & $0.9 \mathrm{E}-03$ \\
\hline 8 & 4 & 6 & 2 & 353601534 & 351174600 & $60 \mathrm{~F}_{0} 03$ \\
\hline 8 & 4 & 0 & 2 & $18.737 \mathrm{sec}$ & $281.547 \mathrm{sec}$ & $0.9 \mathrm{E}-03$ \\
\hline 0 & 1 & 7 & 2 & 363417939 & 361058800 & $653 \mathrm{~F}_{0} 03$ \\
\hline 9 & 1 & 1 & 2 & $17.399 \mathrm{sec}$ & $9408.281 \mathrm{sec}$ & 0.JगE-Uכ \\
\hline 10 & 1 & 9 & 2 & 376355739 & 373590000 & $74 \mathrm{~F}_{-} 03$ \\
\hline 10 & 1 & 9 & 2 & $17.41 \mathrm{sec}$ & $595775.44 \mathrm{sec}$ & $1.4 \mathrm{E}-03$ \\
\hline
\end{tabular}

Table 11. Load of each phase in Ampere.

\begin{tabular}{|c|c|c|c|c|c|}
\hline $\begin{array}{c}\text { Transformer } \\
\text { Capacity }\end{array}$ & Site Number & $\begin{array}{c}\text { Feeder } \\
\text { Number }\end{array}$ & R & S & T \\
\hline $100 \mathrm{KVA}$ & 1 & 1 & 107 & 45 & 132 \\
\hline $200 \mathrm{KVA}$ & 2 & 1 & 153 & 115 & 178 \\
\hline
\end{tabular}


Table 12. Result of solving large-scale problems with GA.

\begin{tabular}{|c|c|c|}
\hline $\begin{array}{c}\text { Selected } \\
\text { path }\end{array}$ & $\begin{array}{c}\text { Amount of electrical } \\
\text { current (Ampere) }\end{array}$ & $\begin{array}{c}\text { Cable } \\
\text { Size } \\
\text { (mm2) }\end{array}$ \\
\hline$[\mathbf{1 - 1 - 1 - 9 ) : [ 1 ]}$ & 107 & 25 \\
\hline $\mathbf{( 9 - 1 )}$ & 82 & 25 \\
\hline $\mathbf{( 1 - 2 )}$ & 32 & 10 \\
\hline$[\mathbf{1 - 1 - 2 - 1 4 ) : [ 1 ]}$ & 45 & 16 \\
\hline$[\mathbf{1 - 1 - 3 - 6 ) : [ 1 ]}$ & 132 & 35 \\
\hline $\mathbf{( 6 - 4 )}$ & 100 & 25 \\
\hline$[\mathbf{2 - 1 - 1 - 1 0 ) : [ 2 ]}$ & 153 & 50 \\
\hline $\mathbf{( 1 0 - 3 )}$ & 121 & 35 \\
\hline $\mathbf{( 3 - 5 )}$ & 96 & 25 \\
\hline$[\mathbf{2 - 1 - 2 - 8 ) : [ 2 ]}$ & 115 & 25 \\
\hline$(\mathbf{8 - 7})$ & 100 & 16 \\
\hline $\mathbf{( 7 - 1 1 )}$ & 50 & 70 \\
\hline$[\mathbf{2 - 1 - 3 - 1 5 ) : [ 2 ]}$ & 178 & 35 \\
\hline $\mathbf{( 1 5 - 1 2 )}$ & 128 & 25 \\
\hline $\mathbf{( 1 2 - 1 3 )}$ & 96 & \\
\hline \multicolumn{2}{|c|}{$\mathbf{5 7 . 0 7 2}$ sec } \\
\hline
\end{tabular}

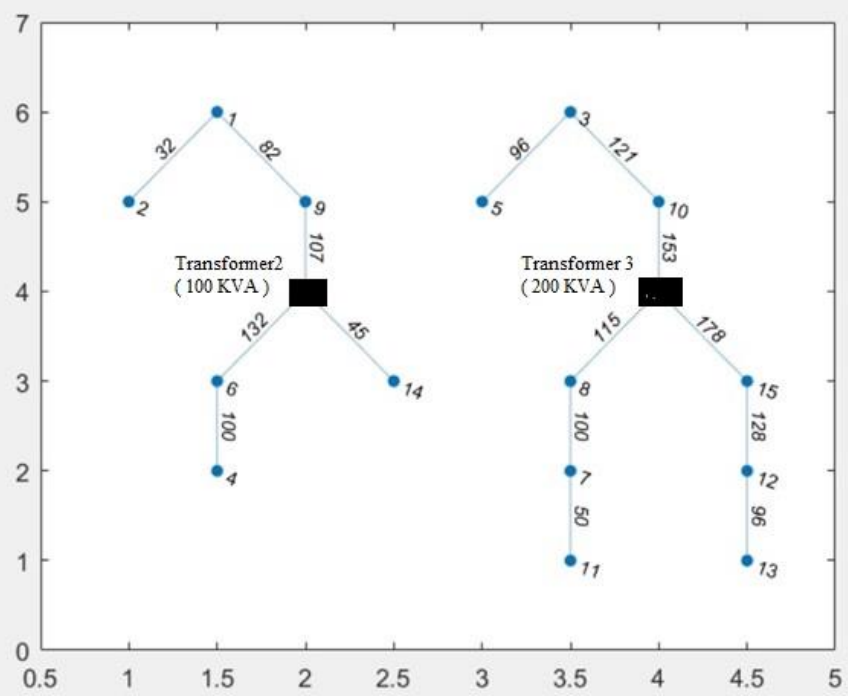

Figure 5. Consumers allocating to the different phases of two transformers 


\section{Conclusion}

An extensive optimization model of power distribution network was presented. In our model, all effective elements on total costs of the power distribution network were designated such as cable sizes, length of feeders, etc. Here, electric current moves from transformers towards the consumption zones. Because of the complexity of power distribution network design, in applying optimization, we formulated a mixed integer programming model for the network. The attained results from the recommended model were promising. Introducing a linear model to design an optimal power distribution network considering different sizes and types of cables and optimal dispositions of distribution transformers was the main contribution of our work.

Here, to minimize the location-allocation costs, an MST method was used. Also, by considering different sizes and types of cables, an optimal cable line system was designed. To make a case study, the actual information on Tehran power distribution company in Iran was applied. By utilizing GAMS 24.7.4 software package, the optimal results were attained. Due to the inability of this software to provide solutions for large size problems in a reasonable time, a genetic algorithm (GA) was proposed. The obtained results of GA were compared with results extracted by the exact method. Numerical results showed the effectiveness of the GA for power distribution network optimization. Finally, attained results encouraged us to investigate networks with more complexities. Here, we assumed that demands of the consumers were single-phase. In future, one may study power distribution networks with a combination of both single-phase and three-phase demands.

\section{References}

[1] Boulaxis, N.G., and Papadpoulos, M. (2002), Optimal feeder routing in distribution system planning using dynamic programming technique and GIS facilities, IEEE Transactions on Power Systems, 17 (1), 242-247.

[2] Fallah, M., Mohajeri, A., and Barzegar-Mohammadi, M. (2016), A new mathematical model to optimize a green gas network using a novel hybird GA/SA algorithm: A case study, IEEE International Conference on Computer and Industrial Engineering (CIE), Tianjin, China, 1142-1149.

[3] Garey, M.R., and Johnson, D.S. (1979), Computers and Intractability: A Guide to the Theory of NP-Completeness, Series of books in Mathematical Sciences, W.H. Freeman.

[4] Gavish, B. (1991), Topological design of telecommunication networks-local access design methods, Annals of Operations Research, 33, 17-71.

[5] Glover, F., Klingman, D., Krishnan, R., and Padman, A. (1992), An in-depth empirical investigation of non-greedy approaches for the minimum spanning tree problem, European Journal of Operational Research, 56, 343-356.

[6] Gomez, J. F., Khodr, H. M., and De Oliviera, P. M. (2004), Ant colony system algorithm for the planning of primary distribution circuits, IEEE Transactions on Power Systems, 19 (2), 996-1004.

[7] Goswami, S (1997), Distribution system planning using branch exchange technique, IEEE Transactions on Power Systems, 12 (2), 718-723.

[8] Graham, R.L., and Hell, P. (1985), On the history of the minimum spanning tree problem, Annals of the History of Computing, 7, 43-57.

[9] Haupt, R. L., and Haupt, S. E. (1998), Practical Genetic Algorithms. New York: WileyInterscience.

[10] Haymond, R.E., Jarvis, J.P., and Shier, D.R. (1984), Computational methods for minimum 
spanning tree algorithms, SIAM Journal on Scientific and Statistical Computing, 5(1), $157-174$.

[11] Koza, J. R. (1994), Introduction to genetic programming, In K. E. Kinnear (Ed.), Advances in Genetic Programming, Cambridge: MIT Press, pp. 21-41.

[12] Li, N., Chen, L., and Low, S. (2012), Demand response in radial distribution networks: distributed algorithm, IEEE International Conference on Smart Grid Communications, pp. 1549-1553.

[13] Maffoli, F. (1981), Complexity of optimum undirected tree problems: a survey of recent results. In: Ausiello G, Lucertini M. editors. Analysis and design of algorithms in combinatorial optimization. International Center for Mechanical Sciences. CISM Courses and Lectures, New York: Springer, 266, pp. 107-128.

[14] Mohajeri, A., Mahdavi, I., and Mahdavi-Amiri, N. (2012a). Optimal pipe diameter sizing in a tree-structured gas network: a case study, International Journal of Industrial and Systems Engineering, 12 (3), 346-368.

[15] Mohajeri, A., Mahdavi, I., Mahdavi-Amiri, N., and Tafazzoli, R. (2012b), Optimization of tree-structured gas distribution network using ant colony optimization: a case study, International Journal of Engineering, 25 (2), 141-158.

[16] Moret, B.M.E., and Shapiro, D. (1991), How to find a minimum spanning tree in practice, In: Maurer H. (editor), New results and new trends in computer science: Proceedings, Graz, Austria, Lecture Notes in Computer Science, 555, pp. 192-203.

[17] Nahman, J. M., and Peric, D. M. (2008), Optimal planning of radial distribution networks by simulated annealing technique, IEEE Transactions On Power Systems, 23 (3), 790-795.

[18] Pierce, A.R. (1956), Bibliography on algorithms for shortest path, shortest spanning treeand related circuit routing problems, Networks, 5 (2), 129-149.

[19] Simon, D. (2013), Evolutionary Optimization Algorithms: Biologically-Inspired and Population Based Approaches to Computer Intelligence, Hoboken, Wiley.

[20] Vamanan, J.F., Wang, Q., Batta, R., and Szczerba, R.J. (2004), Integration of COTS software products ARENA \& CEPLEX for an inventory /logistics problem, Computers \& Operation Research, 31, 533-547.

[21] Zine, R., El Yassini, K., and Rassouli, M. (2011), Multicriteria optimization approach of the electricity distribution planning network problem, Open Problems Compt. Math., 4 (3), 162-176. 